\title{
EVOLVING PHYLOGENIES OF TRAIT-DEPENDENT BRANCHING WITH MUTATION AND COMPETITION PART I: EXISTENCE
}

\author{
SANDRA KLIEM AND ANITA WINTER
}

\begin{abstract}
We propose a type-dependent branching model with mutation and competition for modeling phylogenies of a virus population. The competition kernel depends for any two virus particles on the particles' types, the total mass of the population as well as genetic information available through the number of nucleotide substitutions separating the virus particles. We consider the evolving phylogenies of this individual based model in the huge population, short reproduction time and frequent mutation regime, and show tightness in the state space of marked metric measure spaces. Due to heterogeneity in the natural branching rates, the phylogenies are in general not ultra-metric. We therefore develop new techniques for verifying a compact containment condition. Finally, we characterize the limit as a solution of a martingale problem.
\end{abstract}

Contents

1. Introduction and Motivation

2. The model

2.1. The state space: Marked metric measure spaces

2.2. The trait-dependent branching particle model with mutation and competition

2.3. The fast evolving small mass and large population rescaling

2.4. The martingale problem of the limit dynamics

3. Uniform moment bounds for the discrete models

4. Uniform convergence of generators

5. The compact containment condition

6. Finishing up (Proofs of Theorems 1 and 2

References

\section{Introduction And Motivation}

For many RNA viruses the lack of a proofreading mechanism in the virus' RNA polymerase results in frequent mutation. The high mutation and replication rates cause viral variability which provides one of the main obstacles for the host immune response to control an infection. Therefore it is of great interest to understand in detail the forces which maintain this diversity. In the last decade there has been a growing biology literature describing and classifying the epidemiological and phylogenetic pathogen patterns for particular viruses and bacteria within one host and within the host population (compare, for example, the excellent survey papers

Date: October 20, 2018.

2000 Mathematics Subject Classification. Primary: 60K35, 60J25; Secondary: 60J80, 92D25.

Key words and phrases. tree-valued Markov process, branching process, mutation, selection, competition, martingale problem, marked metric measure space, Gromov-weak topology, compact containment, non-ultrametric, phylogenies.

Research was supported by the DFG through the SPP Priority Programme 1590. 

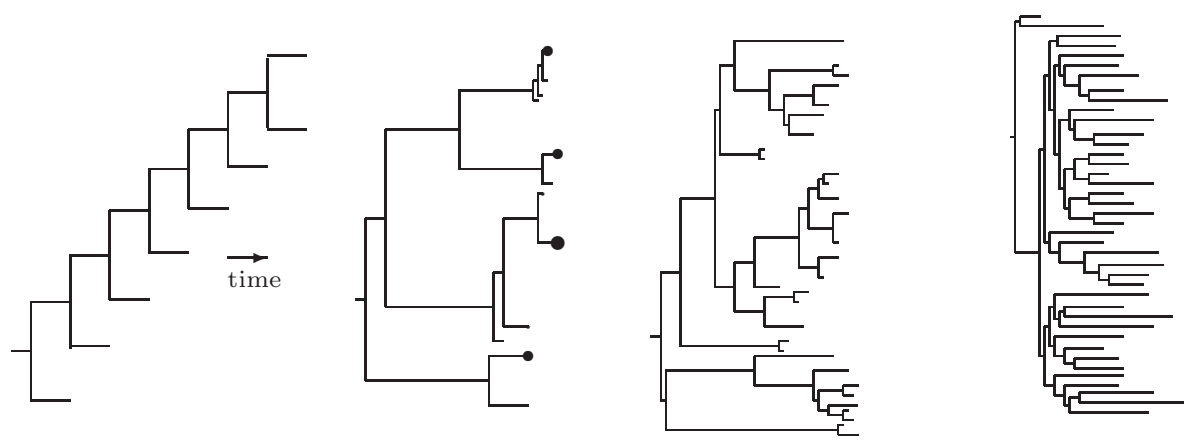

Figure 1. shows a) one dominating strain; b) a bounded number of coexisting strains; c) an unbounded number of coexisting strains with proper frequencies; d) an unbounded number of coexisting strains without proper frequencies. Note that the branch lengths are according to the number of substitutions. (The phylogeny pictures are highly inspired by the figures given in $\left[\mathrm{GPG}^{+} 04.\right)$

$\mathrm{GPG}^{+} 04$, LO07] and the references therein). The patterns range from just a few types at any given time and one dominating type lasting a long time (for instance, influenza on population level or HIV on intra-host level) to many types at any given time but none of them lasting very long (for instance, HIV and $\mathrm{HCV}$ on population level). A simple branching model with selection which features this dichotomy between long and short lasting dominating types has been suggested and further studied in [LS09, BK14].

If one reconstructs the phylogenetic tree based on the number of nucleotide substitutions (compare [BvH99]), then the two situations described above translate into a trivial tree consisting of just one point representing the clan of the only dominating type versus a radial spread outward from a most recent common ancestor. Note that in the first case the tree is often drawn with temporal structure including all the fossils yielding a very skinny tree which consists of a trunk depicting the ancestry of the dominating type and a few short edges reflecting the high rate of extinction (compare the phylogeny class a) in Figure 10. In the latter case of coexisting types, a more elaborate investigation of the patterns suggests to further distinguish between the phylogeny classes b), c) and d) in Figure 1, The main difference between them might be according to statistics such as the degree of balance of a tree, the number of coexisting strains or the asymptotic behaviour of the total length of the subtree spanned by a huge sample.

In this paper and future work we seek to explore the phylogenetic patterns which are primarily affected by natural selection that arises from cross-immunity, that is, the differential effect of immune responses on genetically variable strains. We propose a parametric model for evolving phylogenies which shows these phylodynamic patterns on the level of phylogenies. For that purpose we consider an individual based multi-type branching model with mutation and competition in which virus particles branch with a natural branch rate depending on its trait. Additionally, for each pair of virus particles, a competition kernel allows for either one virus particle killing the other or enhancing it to give birth. The competition kernel might depend on the current population size, on the types of the two competing virus particles as well as their mutual distance (counting the number of substitutions). In this manner the response of the immun system such as cross-immunity is imlicitly taken into account. Newborn virus particles 
are clones of their parent unless mutation occurs. When mutation occurs, the parent particle gives birth to a mutant whose distances to all other virus particles (including its own parent) is set to be "one unit" longer than the corresponding distances from its parent. We are interested in the huge population, fast branching with frequent mutation but small mutation effects limit.

A similar multi-type branching model describing the evolving empirical trait distributions was introduced in DG03]. In that paper the authors even allow for natural branching rates which might also depend on the population size. However, their model is not suitable for modeling cross-immunity as their competition kernel does not take into account any information on the traits' history. Including historical information without loosing the Markov property requires to leave the measure-valued set-up and to work with more enriched state spaces. This issue is resolved in MT12] by working with historical processes.

Historical processes have been established earlier in DP91, GLW05] for structured neutral populations. They provide an ad-hoc coding of the genealogical relationships which is very much intertwined with the spatial interaction, where in the context of multi-type models "spatial" refers to mutation in type space rather than migration in geographic space. That means in particular, that genealogies cannot be read off this way in non-spatial situations. Moreover, Markov processes with values in measures on path-space are notationally far too involved to allow for explicit calculations. We therefore propose to rather encode our multi-type phylogenies as marked metric measure spaces, and restrict ourselves to a dependence of the traits's history only through the phylogenetic distances. This is also closer to applications where the raw data are samples of gene sequences.

The space of marked metric measure spaces equipped with the marked Gromov weak topology has been introduced in [Pio10, DGP11]. It relies on the idea of encoding "spatial trees" as "tree-like" metric spaces which are equipped with a sampling measure, while in addition each point in the tree is assigned a distribution on type-space. It extends the space of (non-spatial) metric measure spaces and the Gromov-weak topology developed in GPW09] (compare also G112, Löh13]).

So far only a few examples of dynamics with values in metric measure spaces can be found in the literature. The first paper which considers dynamics with values in metric (probability) measure spaces is GPW13 and constructs the evolving genealogies of (neutral) resampling dynamics. These were extended into type- and state dependent resampling dynamics (including the Otha-Kimura model) in Pio10 and to resampling dynamics with selection in [DGP12. Note that the dynamics introduced in the latter paper agrees with our dynamics in the case of constant natural branching rates and conditioned on the total mass being a constant. Evolving genealogies of spatially structured resampling populations and their continuum space limit are constructed in GSW16]. In G112 the evolving genealogies of a (neutral) state-dependent branching dynamics (including Feller's branching diffusion and the Anderson model) are considered. It is worthwhile to note that [Gl12] and the present article do not restrict themselves to metric probability measure spaces but allow for arbitrary finite measures. As a result, the occurrences of explosion or extinction of mass now have to be taken into account as well.

Finally notice that metric measure space valued dynamics for evolving phylogenies from a non-evolutionary point of view were already studied in [EW06].

The paper is organized as follows: In Section 2 we introduce our model. We will start with recalling the notion of marked metric measure spaces which we will use for encoding our 
phylogenies. We then introduce the discrete model of evolving phylogenies for a trait-dependent branching particle model with mutation and competition. We further state that if we rescale the virus particle mass, the mutation steps and the length of distance growth in case of mutation appropriately, then we obtain a tight family of stochastic processes. Finally, we represent the tree-valued dynamics arising in the limit as solutions of a martingale problem. We will defer a proof of uniqueness of this martingale problem to a forthcoming paper. Section 3 is devoted to uniform moment estimates which will imply that the discrete model is well-defined by its transition rates and which will be used in the tightness argument. In Section 4 we identify potential limits by means of convergence of generators. In Section 5 we establish the compact containment condition. Due to heterogeneous exponential branching rates, the phylogenies are in general not ultra-metric. We therefore develop new techniques, including sophisticated coupling techniques. The remaining proofs of the results are collected in Section 6 ,

\section{THE MODEL}

In this section we introduce the discrete model of evolving phylogenies of a trait-dependent branching particle model with mutation and competition and its diffusion limit. In Subsection 2.1 we start by presenting and extending results on marked metric measure spaces. The discrete model is introduced in Subsection 2.2. Small individual mass, frequent mutation with small mutation steps and small substitution distance growth in case of mutation is discussed in Subsection 2.3. Finally in Subsection 2.4 we present the diffusion limit.

2.1. The state space: Marked metric measure spaces. In this subsection we define the state space for our evolving phylogenies. The goal is to capture a phylogeny of a multi-type population in such a way that it allows an explicit description of the population size as well as of the ancestral relationships and traits in any finite sample. We will rely on the space of metric measure spaces equipped with the Gromov-weak topology introduced in the context of monotype populations in GPW09] (see also Chapter $3 \frac{1}{2}$ in Gro99]). Its extension to multi-type populations leads to marked metric measure spaces equipped with the marked Gromov-weak topology introduced in DGP11] (compare also Pio10).

As usual, given a topological space $(X, \mathcal{O})$ we denote by $\mathcal{M}_{1}(X)$ and $\mathcal{M}_{f}(X)$ the spaces of probability measures and finite measures on $X$, respectively, defined on the Borel- $\sigma$-algebra of $X$, and by $\Rightarrow$ weak convergence in $\mathcal{M}_{1}(X)$ and $\mathcal{M}_{f}(X)$. Recall that the support $\operatorname{supp}(\mu)$ of $\mu \in \mathcal{M}_{1}(X)$ or $\mu \in \mathcal{M}_{f}(X)$ is the smallest closed set $X_{0} \subseteq X$ such that $\mu\left(X \backslash X_{0}\right)=0$. The push forward of $\mu$ under a measurable map $\varphi$ from $X$ into another topological space $Z$ is the probability measure $\varphi_{*} \mu \in \mathcal{M}_{1}(Z)$ defined by

$$
\varphi_{*} \mu(A):=\mu\left(\varphi^{-1}(A)\right),
$$

for all Borel subsets $A \subseteq Z$. We denote by $\mathcal{B}(X)$ and $\mathcal{C}_{b}(X)$ the bounded real-valued functions on $X$ which are measurable and continuous, respectively.

The states of evolving phylogenies will be marked metric measure spaces, where the marks are elements in the trait space $K$. Throughout the paper we will assume that

$K$ is a complete and separable metric space.

The following definition agrees with Definition 2.1 in [DGP11] (albeit stated there for probability rather than finite measures only). 
Definition 2.1 (mmm-spaces). A K-marked metric measure space (mmm-space, for short) is a triple $(X, r, \mu)$ such that $(X, r)$ is a complete and separable metric space and $\mu \in \mathcal{M}_{f}(X \times K)$, where $X \times K$ is equipped with the product topology.

We call two mmm-spaces $\left(X, r_{X}, \mu_{X}\right)$ and $\left(Y, r_{Y}, \mu_{Y}\right)$ equivalent if they are measure- and mark-preserving isometric, that is there is a measurable $\varphi: \operatorname{supp}\left(\mu_{X}(\cdot \times K)\right) \rightarrow \operatorname{supp}\left(\mu_{Y}(\cdot \times K)\right)$ such that

$$
r_{X}\left(x, x^{\prime}\right)=r_{Y}\left(\varphi(x), \varphi\left(x^{\prime}\right)\right) \text { for all } x, x^{\prime} \in \operatorname{supp}\left(\mu_{X}(\cdot \times K)\right)
$$

and

$$
\tilde{\varphi}_{*} \mu_{X}=\mu_{Y} \text { for } \tilde{\varphi}(x, u)=(\varphi(x), u) .
$$

It is clear that the property of being measure- and mark preserving isometric is an equivalence relation. We write $\overline{(X, r, \mu)}$ for the equivalence class of a mmm-space $(X, r, \mu)$. Define the set of (equivalence classes of) $K$-marked metric measure spaces

$$
\mathbb{M}^{K}:=\{\chi=\overline{(X, r, \mu)}:(X, r, \mu) K \text {-marked metric measure space }\} .
$$

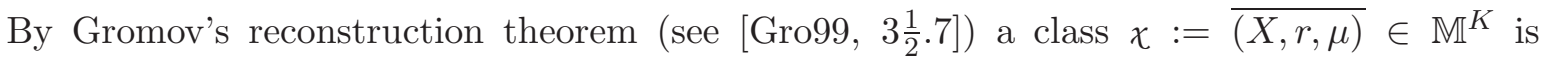
uniquely characterized by the total mass

$$
m(x):=\mu(X \times K)
$$

and the marked distance matrix distribution

$$
\nu^{\chi}:=\left(R^{(X, r), K}\right)_{*} \bar{\mu}^{\otimes \mathbb{N}} \in \mathcal{M}_{1}\left(\mathbb{R}_{+}^{\left(\begin{array}{c}
\mathbb{N} \\
2
\end{array}\right)} \times K^{\mathbb{N}}\right),
$$

with the sampling measure

$$
\bar{\mu}:=\left\{\begin{array}{cc}
\frac{\mu}{m(\chi)}, & m(\chi) \neq 0, \\
\text { arbitrary in } \mathcal{M}_{1}(X \times K), & m(\chi)=0,
\end{array}\right.
$$

and where given a metric space $(X, r)$,

$$
R^{(X, r), K}:= \begin{cases}(X \times K)^{\mathbb{N}} & \rightarrow \mathbb{R}_{+}^{\left(\begin{array}{c}
\mathbb{N} \\
2
\end{array}\right) \times K^{\mathbb{N}}} \\
\left(\left(x_{i}, \kappa_{i}\right)_{i \geq 1}\right) & \mapsto\left(\left(r\left(x_{i}, x_{j}\right)\right)_{1 \leq i<j},\left(\kappa_{i}\right)_{i \geq 1}\right)\end{cases}
$$

denotes the marked distance matrix map. In what follows we abbreviate

$$
\underline{\underline{r}}:=\left(r_{i, j}\right)_{1 \leq i<j}:=\left(r\left(x_{i}, x_{j}\right)\right)_{1 \leq i<j},
$$

and

$$
\underline{\kappa}:=\left(\kappa_{i}\right)_{i \geq 1} .
$$

We base our notion of convergence in $\mathbb{M}^{K}$ on weak convergence of marked distance matrix distributions. This topology was introduced in [DGP11, Definition 2.4] for marked metric probability measure spaces. 
Definition 2.2 (Marked Gromov-weak topology). A sequence $\left(x_{n}\right)_{n \in \mathbb{N}}$ in $\mathbb{M}^{K}$ is said to converge marked Gromov-weakly to $x \in \mathbb{M}$, as $n \rightarrow \infty$, if and only if

$$
m\left(x_{n}\right) \cdot \nu^{x_{n}} \underset{n \rightarrow \infty}{\Longrightarrow} m(\chi) \cdot \nu^{x}
$$

in the weak topology on $\mathcal{M}_{f}\left(\mathbb{R}_{+}^{\left(\begin{array}{c}\mathbb{N} \\ 2\end{array}\right)} \times K^{\mathbb{N}}\right)$. Denote this by $x_{n} \rightarrow \chi$.

Note that we cannot immediately conclude from the fact that the space of finite measures equipped with the weak topology is a Polish space that $\mathbb{M}^{K}$ equipped with the marked Gromovweak topology is Polish. The reason is that the weak limit of a sequence of marked distance matrix distributions is in general not the marked distance matrix distribution of a metric measure space. The following result extents [DGP11, Theorem 2] from marked metric probability to marked metric (finite) measure spaces.

Proposition 2.3 (Polish space). The space $\mathbb{M}^{K}$ equipped with the marked Gromov-weak topology is Polish.

This follows from the extension of the characterization of compact sets given in DGP11, Theorem 3] (compare also [GPW09, Proposition 7.1, Remark 7.2]):

Proposition 2.4 (Relative compactness in $\mathbb{M}^{K}$ ). A family $\Gamma \subset \mathbb{M}^{K}$ is relatively compact if and only if for all $\varepsilon>0$ there exists $N_{\varepsilon} \in \mathbb{N}$ and a compact subset $K_{\varepsilon} \subset K$ such that for all $\chi=\overline{(X, r, \mu)} \in \Gamma$, the following hold:

(i) $m(\chi) \leq N_{\varepsilon}$.

(ii) $\mu\left(X \times K_{\varepsilon}^{c}\right) \leq \varepsilon$.

(iii) There is a subset $X_{\varepsilon} \subseteq X$ with

(iii-a) $\mu\left(X_{\varepsilon}^{c} \times K\right) \leq \varepsilon$,

(iii-b) $X_{\varepsilon}$ has diameter at most $N_{\varepsilon}$, and

(iii-c) $X_{\varepsilon}$ can be covered by at most $N_{\varepsilon}$ balls of radius $\varepsilon$.

Proof of Proposition 2.3 and 2.4. This follows from Theorem 3 in DGP11 together with the fact that $x_{n} \rightarrow \chi$ if and only if $m\left(\chi_{n}\right) \rightarrow m(\chi)$ and $\nu^{\chi_{n}} \underset{n \rightarrow \infty}{\Longrightarrow} \nu^{\chi}$ in case $m(\chi) \neq 0$, or equivalently, if $m\left(\chi_{n}\right) \rightarrow m(\chi)$ and $m(\chi) \nu^{\chi_{n}} \underset{n \rightarrow \infty}{\Longrightarrow} m(\chi) \nu^{\chi}$.

2.2. The trait-dependent branching particle model with mutation and competition. In this subsection we introduce our individual based model in detail.

Fix a distance constant $\ell>0$ and an individual mass constant $\zeta>0$. The trait-dependent branching particle model with mutation and competition takes values in phylogenies of a finite population in which each individual has mass $\zeta>0$ and substitution distances are assumed to be multiples of $\ell$ (compare Figure 2.2).

The state space is therefore the subspace of $(\ell, \zeta)$-marked metric measure spaces defined as

$$
\begin{aligned}
\mathbb{M}^{K,(\ell, \zeta)}:=\left\{x=\overline{(X, r, \mu)} \in \mathbb{M}^{K}:\right. & \\
& \zeta^{-1} \mu(\cdot \times K) \in \mathcal{N}_{f}(X) ; \# \operatorname{supp}(\mu(\{x\} \times \cdot)) \in\{0,1\}, \forall x \in X, \\
& \left.\ell^{-1} r\left(x, x^{\prime}\right) \in \mathbb{N} \cup\{0\}, \forall x, x^{\prime} \in X\right\},
\end{aligned}
$$

where $\mathcal{N}_{f}(X)$ denotes the space of non-negative integer-valued measures on $X$. 
Remark 2.5 (Mark functions for finite populations). Notice that if $\chi \in \mathbb{M}^{K,(\ell, \zeta)}$ there exists a measurable mark function $\tilde{\kappa}: X \rightarrow K$ such that

$$
\mu(\mathrm{d}(x, \kappa))=\mu(\mathrm{d} x \times K) \otimes \delta_{\tilde{\kappa}(x)}(\mathrm{d} \kappa) .
$$

In Section 2.3 it is stated that a suitably rescaled family of tree-valued $\left(\ell_{N}, \zeta_{N}, \alpha_{N}\right)$-traitdependent branching dynamics $\mathcal{X}^{N} \in \mathbb{M}^{K,\left(\ell_{N}, \zeta_{N}\right)}, N \in \mathbb{N}$ with mutation and competition is tight in $\mathbb{M}^{K}$. It remains as an open question, whether all subsequential limits admit a mark function. As a first step in this direction, a criterion for the existence of a mark function has been derived in KL15] and successfully been applied to the tree-valued Fleming-Viot dynamics with mutation and selection from [DGP12]. This criterion has been extended to marked metric boundedly finite measure spaces and then applied to the spatially interacting Fleming-Viot dynamics on $\mathbb{Z}$ in GSW16. Furthermore, in [GSW16] a version of the continuum limit of these spatially interacting tree-valued Fleming-Viot dynamics with a mark function is constructed by means of the Brownian web.

Remark 2.6 (Link between $(\ell, \zeta)$-marked metric measure spaces and evolving phylogenies). If $(X, r, \mu)$ is such that $r$ is only a pseudo-metric on $X$, (that is $r(x, y)=0$ is possible for $x \neq y$ ) we can still define its measure-preserving isometry class under the additional assumption that if $r(x, y)=0$, then $\mu(\{x\} \times \cdot)=\mu(\{y\} \times \cdot)$. Since this class contains also metric measure spaces, there is a bijection between the set of equivalence classes of pseudo-metric measure spaces and the set of equivalence classes of metric measure spaces and we will use both notions interchangeably.

It turns out that this is particularly suited to our set-up. Given a pseudo-metric measure space $(X, r, \mu)$, we would like to think of two individuals $x \neq y \in X$ with $\mu(\{x\} \times K) \cdot \mu(\{y\} \times K)>0$ but $r(x, y)=0$ as clones (of each other). In the corresponding equivalent metric measure space they are all collected into one clan (of clones). If $\chi=\overline{(X, r, \mu)} \in \mathbb{M}^{K,(\ell, \zeta)}$, we can recover the number of clones in clan $x \in X$ as

$$
n_{x}:=\frac{1}{\zeta} \cdot \mu(\{x\} \times K),
$$

as $\zeta$ stands for an individual's mass. In particular,

$$
n:=\sum_{x \in X} n_{x}=\frac{m(x)}{\zeta}
$$

relates the total number of individuals in the population with the total mass.

To be in a position to introduce the discrete model, fix $p \in[0,1]$ which we refer to as the mutation probability. Moreover, let $\alpha$ be a stochastic kernel on $K$. In the following we refer to $\alpha$ as the mutation kernel. We will exclude the possibility of non-trivial mutation, that is, we assume for every $\kappa \in K$,

$$
\alpha(\kappa,\{\kappa\})=0 .
$$

Further let $\beta: K \rightarrow[0, \infty), \gamma^{\text {birth }}: \mathbb{R}_{+} \times \mathbb{R}_{+} \times K^{2} \rightarrow[0, \infty)$ and $\gamma^{\text {death }}: \mathbb{R}_{+} \times \mathbb{R}_{+} \times K^{2} \rightarrow[0, \infty)$.

The trait-dependent branching particle model with mutation and competition is a Markov process which takes values in $\mathbb{M}^{K,(\ell, \zeta)}$. Given that it starts in $\chi_{0} \in \mathbb{M}^{K,(\ell, \zeta)}$, it has the following dynamics: 


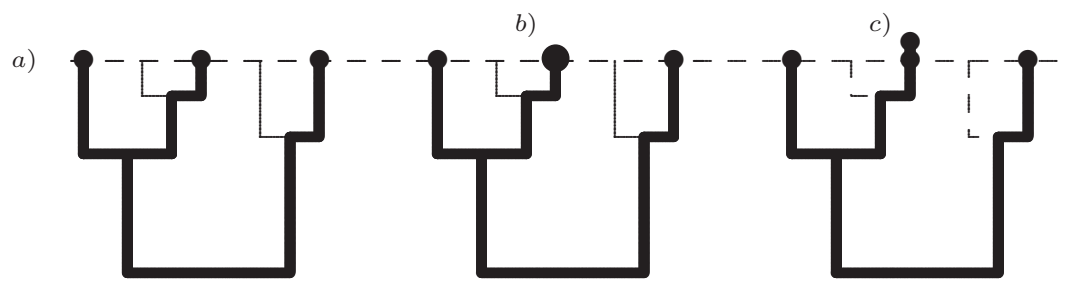

FIGURE 2. shows the phylogeny of a sample of a phylogeny of sample size 3, a) before the branching event, and after the second individual gave birth to b) a clone (indicated by the weight enlarged by $\zeta$ on the clan containing this clone) or c) a mutant (indicated by the mutant child being in positive genetic distance $\ell$ to its mother).

- Death. For any particle from clan $x_{1}$ of trait $\kappa_{1}=\tilde{\kappa}\left(x_{1}\right)$ and any particle from clan $x_{2}$ of trait $\kappa_{2}=\tilde{\kappa}\left(x_{2}\right)$, at rate

$$
\frac{\beta\left(\kappa_{2}\right)}{m(\chi)}+\zeta \cdot \frac{\gamma^{\text {death }}\left(m(x), r\left(x_{1}, x_{2}\right), \kappa_{1}, \kappa_{2}\right)}{m(\chi)},
$$

the second particle dies either due to natural death or because it gets killed by the first particle. That is, the total death rate for a particle of clan $x_{2}$ and trait $\kappa_{2}=\tilde{\kappa}\left(x_{2}\right)$ is

$$
\begin{aligned}
& \frac{\beta\left(\kappa_{2}\right)}{\zeta}+\sum_{x_{1} \in X} \frac{n_{x_{1}}}{n} \gamma^{\text {death }}\left(m(\chi), r\left(x_{1}, x_{2}\right), \tilde{\kappa}\left(x_{1}\right), \kappa_{2}\right) \\
& =\frac{\beta\left(\kappa_{2}\right)}{\zeta}+\sum_{x_{1} \in X} \bar{\mu}\left(\left\{x_{1}\right\} \times K\right) \gamma^{\text {death }}\left(m(\chi), r\left(x_{1}, x_{2}\right), \tilde{\kappa}\left(x_{1}\right), \kappa_{2}\right) .
\end{aligned}
$$

Such a death event yields the following transition:

$$
\chi \mapsto\left(X, r, \mu-\zeta \cdot \delta_{\left(x_{2}, \kappa_{2}\right)}\right) .
$$

Notice that if $\zeta^{-1} \cdot \mu\left(\left\{x_{2}\right\} \times K\right)=1$, then $\left(X, r, \mu-\zeta \cdot \delta_{\left(x_{2}, \kappa_{2}\right)}\right)$ is equivalent to $(X \backslash$ $\left.\left\{x_{2}\right\}, r, \mu-\zeta \cdot \delta_{\left(x_{2}, \kappa_{2}\right)}\right)$.

- Birth. For any particle from clan $x_{1}$ of trait $\kappa_{1}=\tilde{\kappa}\left(x_{1}\right)$ and any particle from clan $x_{2}$ of trait $\kappa_{2}$, at rate

$$
\frac{\beta\left(\kappa_{2}\right)}{m(\chi)}+\zeta \cdot \frac{\gamma^{\mathrm{birth}}\left(m(\chi), r\left(x_{1}, x_{2}\right), \kappa_{1}, \kappa_{2}\right)}{m(\chi)}
$$

the second particle gives birth either naturally or due to birth-enhancement by the first particle. That is, the total birth rate of a particle from clan $x_{2}$ of trait $\kappa_{2}$ is

$$
\begin{aligned}
& \frac{\beta\left(\kappa_{2}\right)}{\zeta}+\sum_{x_{1} \in X} \frac{n_{x_{1}}}{n} \gamma^{\operatorname{birth}}\left(m(\chi), r\left(x_{1}, x_{2}\right), \tilde{\kappa}\left(x_{1}\right), \kappa_{2}\right) \\
& =\frac{\beta\left(\kappa_{2}\right)}{\zeta}+\sum_{x_{1} \in X} \bar{\mu}\left(\left\{x_{1}\right\} \times K\right) \gamma^{\operatorname{birth}}\left(m(\chi), r\left(x_{1}, x_{2}\right), \tilde{\kappa}\left(x_{1}\right), \kappa_{2}\right) .
\end{aligned}
$$

With probability $p \in[0,1]$ the newborn $z \notin X$ is a mutant of its parent $x_{2}$ whose new type $\tilde{\kappa}(z)$ is chosen with respect to $\alpha\left(\kappa_{2}, \cdot\right)$, and its distance to all other particles is given by

$$
r^{\left(x_{2}, z\right), \ell}(z, x):=\left\{\begin{aligned}
r\left(x_{2}, x\right)+\ell, & x \in X \\
0, & x=z
\end{aligned}\right.
$$


while with probability $1-p$ the newborn is just a clone of its parent. Under our assumption (2.16) on non-trivial mutation, this yields the following transition:

$$
\chi \mapsto\left(X \uplus\{z\}, r^{\left(x_{2}, z\right), \ell}, \mu+\zeta \cdot \delta_{(z, \tilde{\kappa}(z))} \cdot \mathbf{1}\left\{\tilde{\kappa}(z) \neq \kappa_{2}\right\}+\zeta \cdot \delta_{\left(x_{2}, \kappa_{2}\right)} \cdot \mathbf{1}\left\{\tilde{\kappa}(z)=\kappa_{2}\right\}\right),
$$

where $\tilde{\kappa}(z)$ is chosen with respect to

$$
\widehat{\alpha}_{N}\left(\kappa_{2}, \cdot\right):=p \cdot \alpha\left(\kappa_{2}, \cdot\right)+(1-p) \cdot \delta_{\kappa_{2}}(\cdot) .
$$

We remark that if there is no mutation, $\left(X \uplus\{z\}, r^{\left(x_{2}, z\right), \ell}, \mu+\zeta \cdot \delta_{\left(x_{2}, \kappa_{2}\right)}\right)$ is equivalent to $\left(X, r^{\left(x_{2}, z\right)}, \mu+\zeta \cdot \delta_{\left(x_{2}, \kappa_{2}\right)}\right)$. In this case, the clan $\{z\}$ cannot effect new birth events, as there are no particles in clan $z$ due to $n_{z}=0$ respectively $\mu(\{z\} \times K)=0$.

Notice that the jump process described above is well-defined if we can exclude the explosion of the total rate in finite time. To ensure this we make the following assumptions on the branching and the competition rates.

Assumption 1 (Bounded branching rate). The function $\beta: K \rightarrow[0, \infty)$ is measurable and bounded, that is, there is a constant $\bar{\beta}<\infty$ such that

$$
\sup _{\kappa \in K} \beta(\kappa) \leq \bar{\beta} .
$$

Assumption 2 (Bounded birth-enhancement rate). The function $\gamma^{\text {birth }}: \mathbb{R}_{+} \times \mathbb{R}_{+} \times K^{2} \rightarrow[0, \infty)$ is measurable and bounded, that is, there is a constant $\bar{\gamma}_{b}<\infty$ such that

$$
\sup _{m, r \in \mathbb{R}_{+}, \kappa, \kappa^{\prime} \in K} \gamma^{\text {birth }}\left(m, r, \kappa, \kappa^{\prime}\right) \leq \bar{\gamma}_{b} \text {. }
$$

Assumption 3 (Bounded death-competition rate). There is a continuous function $\tilde{\gamma}: \mathbb{R}_{+} \rightarrow$ $[0, \infty)$ such that for all $m \in \mathbb{R}_{+}$, the function $\gamma^{\text {death }}: \mathbb{R}_{+} \times \mathbb{R}_{+} \times K^{2} \rightarrow[0, \infty)$ is measurable and satisfies

$$
\sup _{r \in \mathbb{R}_{+}, \kappa, \kappa^{\prime} \in K} \gamma^{\text {death }}\left(m, r, \kappa, \kappa^{\prime}\right) \leq \tilde{\gamma}(m)
$$

Under these assumptions the total jump rate can be upper bounded by a continuous function of the total mass, which itself can be stochastically upper bounded by a Yule process with rate $\zeta^{-2} \bar{\beta}+\zeta^{-1} \bar{\gamma}_{b}$. We can therefore immediately conclude the following:

Proposition 2.7 (Well-posed). Under Assumptions 1, : 2 and [3, there is a well-defined strong Markov pure jump process, $\mathcal{X}=\left(\mathcal{X}_{t}\right)_{t \geq 0}$, with the above described transition rates provided we start in a random $\mathcal{X}_{0} \in \mathbb{M}^{K,(\ell, \zeta)}$ with $\mathbb{E}\left[m\left(\mathcal{X}_{0}\right)\right]<\infty$.

This leads to the following definition.

Definition $2.8((\ell, \zeta, \alpha)$-trait-dependent branching with mutation and competition). The process $\mathcal{X}=\left(\mathcal{X}_{t}\right)_{t \geq 0}$ (starting in $\mathcal{X}_{0}$ with $\left.\mathbb{E}\left[m\left(\mathcal{X}_{0}\right)\right]<\infty\right)$ is referred to as tree-valued $(\ell, \zeta, \alpha)$-traitdependent branching dynamics with mutation and competition. 
2.3. The fast evolving small mass and large population rescaling. In this subsection we state that the suitably rescaled family of tree-valued $(\ell, \zeta, \alpha)$-trait-dependent branching dynamics with mutation and competition is tight.

We are interested in a rescaling where the particles branch fast and a mutation event is a typical event while the mutation steps are small. This translates into letting the parameters $\zeta$ and $\ell$ tend to zero such that the fraction of $\ell$ and $\zeta$ converges to a non-trivial limit. Note that the mutation parameter $p$ is kept constant. For simplicity, we choose for every $N \in \mathbb{N}$,

- Small distance constant. $\ell_{N}:=\frac{1}{N}$.

- Small individual mass. $\zeta_{N}:=\frac{1}{N}$.

We also make the following assumption.

Assumption 4 (Mutation operator). Consider a family $\left\{\alpha_{N} ; N \in \mathbb{N}\right\}$ of stochastic kernels on $K$ such that the following holds:

(i) Mutation processes along one line are tight. Let for fixed $N \in \mathbb{N}$, $k^{N}$ be the $K$-valued Markov process which jumps at rate $N \cdot \alpha_{N}(\kappa, \mathrm{d} \tilde{\kappa})$ from $\kappa$ to $\tilde{\kappa}$. The family of jump processes $\left\{K^{N} ; N \in \mathbb{N}\right\}$ is assumed to be tight.

(ii) Limit mutation along one line is uniquely characterized. There is a linear operator $(A, \mathcal{D}(A))$ on $\mathcal{C}_{b}(K)$ such that $\mathcal{D}(A)$ is an algebra, dense in $\mathcal{C}_{b}(K)$ and the $(A, \mathcal{D}(A))$-martingale problem has a unique solution, and for all $h \in \mathcal{D}(A)$ and $\kappa \in K$,

$$
N \cdot \int_{K}\left(\alpha_{N}(\kappa, \mathrm{d} \tilde{\kappa})-\delta_{\kappa}(\mathrm{d} \tilde{\kappa})\right) h(\tilde{\kappa}) \underset{N \rightarrow \infty}{\longrightarrow} A h(\kappa),
$$

uniformly in $\kappa \in K$. Here, as usual, $\delta_{\kappa}(\cdot)=\delta(\kappa, \cdot)$ denotes the dirac measure. Note that (2.28) implies that $\int_{K} \alpha_{N}(\cdot, \mathrm{d} \tilde{\kappa}) f(\tilde{\kappa}) \underset{N \rightarrow \infty}{\longrightarrow} f$ whenever $f$ is in $\mathcal{D}(A)$.

We make the following further assumptions on the natural birth and the death-competition rate.

Assumption 5 (Natural birth rate bounded away from zero). There exists a constant $\underline{\beta}>0$ such that

$$
\inf _{\kappa \in K} \beta(\kappa) \geq \underline{\beta}
$$

Remark 2.9. We point out that Assumptions 2 and 5 imply that there exist a $C \in(0, \infty)$ such that for all $\kappa_{2} \in K$,

$$
\sup _{m \in \mathbb{R}_{+}, r \in \mathbb{R}_{+}, \kappa_{1} \in K} \gamma^{\operatorname{birth}}\left(m, r, \kappa_{1}, \kappa_{2}\right) \leq C \cdot \beta\left(\kappa_{2}\right) .
$$

Assumption 6 (Linear bound on the competition death rate). There exists a constant $\bar{\gamma}_{d}$ such that

$$
\sup _{m, r \in \mathbb{R}_{+}, \kappa, \kappa^{\prime} \in K} \gamma^{\text {death }}\left(m, r, \kappa, \kappa^{\prime}\right) \leq(1 \vee m) \bar{\gamma}_{d}
$$

Our first main result is the following: 
Theorem 1 (Tightness). Let for each $N \in \mathbb{N}, \mathcal{X}^{N}$ be the tree-valued $\left(\ell_{N}, \zeta_{N}, \alpha_{N}\right)$-trait-dependent branching dynamics with mutation and competition such that $\left\{\mathcal{X}_{0}^{N} ; N \in \mathbb{N}\right\}$ is a tight family in $\mathbb{M}^{K}$ with $\sup _{N \in \mathbb{N}} \mathbb{E}\left[\left(m\left(\mathcal{X}_{0}^{N}\right)\right)^{3}\right]<\infty$. Under Assumptions 1, 2, 4. 5 and [6, the family $\left\{\mathcal{X}_{N} ; N \in \mathbb{N}\right\}$ is tight.

Definition 2.10 (Tree-valued trait-dependent branching with mutation and competition). Any limit process is called tree-valued trait-dependent branching dynamics with mutation and competition.

2.4. The martingale problem of the limit dynamics. In this subsection we present an analytic representation of the limit process in terms of a martingale problem.

We begin by introducing a class of suitable test functions.

Definition 2.11 (Polynomials). A polynomial is a function $F=F^{h}: \mathbb{M}^{K} \rightarrow \mathbb{R}$ of the form

$$
F(\chi):=\int_{\mathbb{R}_{+}^{\left(\frac{\mathbb{N}}{2}\right)} \times K^{\mathbb{N}}} \nu^{\chi}(\mathrm{d}(\underline{\underline{r}}, \underline{\kappa})) h(m(\chi), \underline{\underline{r}}, \underline{\kappa})
$$

for a function $h \in \mathcal{C}_{b}\left(\mathbb{R}_{+} \times \mathbb{R}_{+}^{\left(\begin{array}{c}\mathbb{N} \\ 2\end{array}\right)} \times K^{\mathbb{N}}\right)$ such that there is a constant $\bar{h}_{0} \in \mathbb{R}$ such that

$$
h(0, \cdot, \cdot) \equiv \bar{h}_{0} .
$$

If the function $h \in \mathcal{C}_{b}\left(\mathbb{R}_{+} \times \mathbb{R}_{+}^{\left(\begin{array}{c}\mathbb{N} \\ 2\end{array}\right)} \times K^{\mathbb{N}}\right)$ depends on $\left(m,\left(r_{i, j}\right)_{1 \leq i<j},\left(\kappa_{i}\right)_{i \in \mathbb{N}}\right)$ only through $\left(m,\left(r_{i, j}\right)_{1 \leq i<j \leq n},\left(\kappa_{i}\right)_{i \leq n}\right)$ for some $n \in \mathbb{N}$, then we refer to $F$ as a polynomial of finite degree.

Denote by $\Pi$ the space of all polynomials, and by $\Pi_{\text {finite }}$ the subspace of polynomials of finite degree.

Recall the mutation operator $(A, \mathcal{D}(A))$ from Assumption 4 . We consider for each $l_{1}, l_{2} \in \mathbb{N}$ the subspace

$$
\begin{array}{r}
\Pi_{0}^{l_{1}, l_{2}, A}:=\left\{F=F^{h} \in \Pi: h(\cdot, \underline{\underline{r}}, \underline{\kappa}) \in \mathcal{C}_{b}^{l_{1}}\left(\mathbb{R}_{+}\right), \forall(\underline{\underline{r}}, \underline{\kappa}) ; h(m, \cdot, \underline{\kappa}) \in \mathcal{C}_{b}^{l_{2}}\left(\mathbb{R}_{+}^{\left(\begin{array}{c}
\mathbb{N} \\
2
\end{array}\right)}\right), \forall(m, \underline{\kappa}) ;\right. \\
\left.h\left(m, \underline{\underline{r}},\left(\kappa_{1}, \ldots, \kappa_{l-1}, \cdot, \kappa_{l+1}, \ldots\right)\right) \in \mathcal{D}(A), \forall l \in \mathbb{N},(m, \underline{\underline{r}}, \underline{\kappa})\right\} .
\end{array}
$$

Recall the branching rate $\beta: K \rightarrow \mathbb{R}_{+}$and the competition rates $\gamma^{\text {birth }}, \gamma^{\text {death }}: \mathbb{R}_{+} \times \mathbb{R}_{+} \times$ $K^{2} \rightarrow \mathbb{R}_{+}$, and put

$$
\Gamma\left(m(\chi), r_{1,2}, \kappa_{1}, \kappa_{2}\right):=\gamma^{\text {birth }}\left(m(\chi), r_{1,2}, \kappa_{1}, \kappa_{2}\right)-\gamma^{\text {death }}\left(m(\chi), r_{1,2}, \kappa_{1}, \kappa_{2}\right) .
$$

In Proposition 4.4 the following operator is obtained as the limit of a sequence of operators corresponding to the suitably rescaled approximating individual based models (cf. Subsections 2.2 and 2.3, and (4.1)). Consider the operator $\Omega$ acting on

$$
\mathcal{D}(\Omega):=\left\{F \in \Pi^{2,1, A}: \Omega F \text { is well-defined and finite }\right\},
$$

where

$$
\Omega:=\Omega_{\text {total mass }}^{\beta, \Gamma}+\Omega_{\text {trait mutation }}^{p, \beta, A}+\Omega_{\text {growth }}^{p, \beta}+\Omega_{\Gamma-\text { reweigh }}^{\Gamma}+\Omega_{\text {natural branching }}^{\beta}
$$

is reflecting 
(1) $\Omega_{\text {total mass }}^{\beta, \Gamma}$ the changes in the total mass due to competition, fluctuation and a flow due to reweighing the sampling measure with respect to updating $\beta$,

(2) $\Omega_{\text {trait mutation }}^{p, \beta, A}$ trait mutation,

(3) $\Omega_{\text {growth }}^{p, \beta}$ growth of substitution distances,

(4) $\Omega_{\Gamma \text {-reweigh }}^{\Gamma}$ a flow due to reweighing the sampling measure with respect to updating $\Gamma$, and

(5) $\Omega_{\text {natural branching }}^{\beta}$ the effects on genealogies spanned by a sample of fixed size due to neutral branching without mutation.

If $m(\chi)=0$, we put $\Omega_{\text {total mass }}^{\beta, \Gamma} F^{h}(\chi)=0$. Notice that this implies that also $\Omega F^{h}(\chi)=0$ as $h(0, \cdot, \cdot)$ is assumed to be constant.

Otherwise, if $m(\chi)>0$ we introduce the several parts step by step. We use the abbreviations:

$$
\widehat{\beta}^{\chi}:=\int \nu^{\chi}(\mathrm{d}(\underline{\underline{r}}, \underline{\kappa})) \beta\left(\kappa_{1}\right)
$$

and

$$
\widehat{\gamma}^{\text {death } / \text { birth }}(m(\chi)):=\int \nu^{\chi}(\mathrm{d}(\underline{\underline{r}}, \underline{\kappa})) \gamma^{\text {death } / \text { birth }}\left(m(\chi), r_{1,2}, \kappa_{1}, \kappa_{2}\right) .
$$

Moreover, we put

$$
\widehat{\Gamma}(m(\chi)):=\int \nu^{\chi}(\mathrm{d}(\underline{\underline{r}}, \underline{\kappa})) \Gamma\left(m(\chi), r_{1,2}, \kappa_{1}, \kappa_{2}\right)
$$

Step 1 (Changes in the total mass). Put

$$
\begin{aligned}
\Omega_{\text {total mass }}^{\beta, \Gamma} F^{h}(\chi)= & : \Omega_{\text {competition }}^{\Gamma} F^{h}(\chi)+\Omega_{\text {total mass fluctuation }}^{\beta} F^{h}(\chi)+\Omega_{\text {mass flow }}^{\beta} F^{h}(\chi) \\
= & \widehat{\Gamma}(m(\chi)) \cdot m(\chi) \cdot \frac{\partial}{\partial m} F^{h}(\chi)+\widehat{\beta}^{\chi} \cdot m(\chi) \cdot \frac{\partial^{2}}{\partial^{2} m} F^{h}(\chi) \\
& +2 \cdot \int \nu^{\chi}(\mathrm{d}(\underline{\underline{r}}, \underline{\kappa})) \frac{\partial}{\partial m} h(m(\chi), \underline{\underline{r}}, \underline{\kappa}) \cdot \sum_{l \geq 1}\left(\beta\left(\kappa_{l}\right)-\widehat{\beta}^{\chi}\right) .
\end{aligned}
$$

In words, given the evolution of the sampling measure, the total mass follows a branching diffusion with branching rate $\widehat{\beta}^{x}$ and state dependent $\operatorname{drift} \widehat{\Gamma}(m(\chi))$. In addition, as $\beta$ is trait dependent, changes in the sampling measure lead to a flow of mass which we would not see if the (natural) branching $\beta$ were a constant.

Step 2 (Trait Mutation). Put

$$
\Omega_{\text {trait mutation }}^{p, \beta, A} F^{h}(\chi)=p \cdot \int \nu^{\chi}(\mathrm{d}(\underline{\underline{r}}, \underline{\kappa})) \sum_{l \geq 1} \beta\left(\kappa_{l}\right) \cdot A^{(l)} h(m(\chi), \underline{\underline{r}}, \underline{\kappa}),
$$

where $A^{(l)}$ acts on $h$ as the mutation operator $A$ on the function of the $l^{\text {th }}$-trait-coordinate of $h$ (assuming that all other variables are kept constant).

Step 3 (Growth of genetic distances). Put

$$
\Omega_{\text {growth }}^{p, \beta} F^{h}(\chi)=p \cdot \int \nu^{\chi}(\mathrm{d}(\underline{\underline{r}}, \underline{\kappa})) \sum_{1 \leq l_{1}<l_{2}}\left(\beta\left(\kappa_{l_{1}}\right)+\beta\left(\kappa_{l_{2}}\right)\right) \cdot \frac{\partial}{\partial r_{l_{1}, l_{2}}} h(m(\chi), \underline{\underline{r}}, \underline{\kappa}) .
$$


That is, the distance between two individuals, one from the clan $x_{1}$ and of current type $\kappa_{1}$ and the other one from the clan $x_{2}$ and of current type $\kappa_{2}$ grows at speed $p \cdot\left(\beta\left(\kappa_{1}\right)+\beta\left(\kappa_{2}\right)\right)$, which indeed models growing of the substitution distance.

Step 4 (Reweigh of the sampling measure with respect to $\Gamma$ ). Put

$$
\left.\Omega_{\Gamma \text {-reweigh }}^{\Gamma} F^{h}(\chi)=\int \nu^{\chi}(\mathrm{d}(\underline{\underline{r}}, \underline{\kappa})) \sum_{l \geq 1}\left(\Gamma\left(m(\chi), r_{1, l+1}, \kappa_{1}, \kappa_{l+1}\right)\right)-\widehat{\Gamma}(m(\chi))\right) \cdot h\left(m, \tau_{1}(\underline{\underline{r}}, \underline{\kappa})\right),
$$

where for $\ell \geq 1, \tau_{\ell}$ denotes an index shift by $\ell \in \mathbb{N}$, that is,

$$
\tau_{\ell}(\underline{\underline{r}}, \underline{\kappa}):=\left(\left(r_{i, j}\right)_{l+1 \leq i<j},\left(\kappa_{i}\right)_{l+1 \leq i}\right) .
$$

Step 5 (Effect of neutral branching without mutation on the genealogy). Put $(2.46)$

$$
\begin{aligned}
& \Omega_{\text {natural branching }}^{\beta} F^{h}(\chi) \\
&=: \Omega_{\beta \text {-reweigh }}^{\beta} F^{h}(\chi)+\Omega_{\text {resample }}^{\beta} F^{h}(\chi) \\
&= \frac{1}{m(\chi)} \int \nu^{\chi}(\mathrm{d}(\underline{\underline{r}}, \underline{\kappa})) \sum_{1 \leq l}\left(\widehat{\beta}^{\chi}-\beta\left(\kappa_{l}\right)\right) h(m(\chi), \underline{\underline{r}}, \underline{\kappa}) \\
&+\frac{1}{m(\chi)} \int \nu^{\chi}(\mathrm{d}(\underline{\underline{r}}, \underline{\kappa})) \sum_{1 \leq l_{1}, l_{2}}\left\{\beta\left(\kappa_{l_{1}}\right)\left(\Theta_{l_{1}, l_{2}} h(m(\chi), \underline{\underline{r}}, \underline{\kappa})-h(m(\chi), \underline{\underline{r}}, \underline{\kappa})\right)+\left(\widehat{\beta}^{\chi}-\beta\left(\kappa_{l_{2}}\right)\right) h(m(\chi), \underline{\underline{r}}, \underline{\kappa})\right\},
\end{aligned}
$$

where for $1 \leq l_{1} \neq l_{2}$, the replacement map $\Theta_{l_{1}, l_{2}}$ on $\mathcal{C}_{b}\left(\mathbb{R}_{+} \times \mathbb{R}_{+}^{\left(\begin{array}{c}\mathbb{N} \\ 2\end{array}\right)} \times K^{\mathbb{N}}\right)$ sends a function to a new function by "replacing its $l_{2}^{\text {nd }}$ argument by its $l_{1}^{\text {st }}$, that is, $\Theta_{l_{1}, l_{2}} h=h \circ \vartheta_{l_{1}, l_{2}}$, where

$$
\vartheta_{l_{1}, l_{2}}\left(m(\chi),\left(r_{i, j}\right)_{1 \leq i<j},\left(\kappa_{i}\right)_{i \in \mathbb{N}}\right):=\left(m(\chi),\left(r_{\theta_{l_{1}, l_{2}}(i), \theta_{l_{1}, l_{2}}(j)}\right)_{1 \leq i<j},\left(\kappa_{\theta_{l_{1}, l_{2}}(i)}\right)_{i \in \mathbb{N}}\right),
$$

and

$$
\theta_{l_{1}, l_{2}}(i):= \begin{cases}i, & \text { if } i \neq l_{2} \\ l_{1}, & \text { if } i=l_{2}\end{cases}
$$

Remark 2.12 (Consistency). We point out that

$$
\Pi^{2,1, A} \cap \Pi_{\text {finite }} \subseteq \mathcal{D}(\Omega) .
$$

More precisely, summands associated with an index $l$ appearing at any of the right hand sides in (2.41)-(2.46) are zero (and thus do not contribute to the series) if the function $h$ does not depend explicitly on the metric entries $r_{i \wedge l, i \vee l}, i \in \mathbb{N}$, and the type entry $\kappa_{l}$. This is obvious to see for all parts of the generator except maybe $\Omega_{\text {resample }}^{\beta}$. We therefore want to present a short argument here. 
Assume first that $l_{1} \in \mathbb{N}$ is such that the function $h$ does not depend on $r_{i \wedge l_{1}, i \vee l_{1}}$ for all $i \in \mathbb{N}$ and also does not depend on $\kappa_{l_{1}}$. In that case,

$$
\begin{aligned}
& \int \nu^{\chi}(\mathrm{d}(\underline{\underline{r}}, \underline{\kappa}))\left\{\beta\left(\kappa_{l_{1}}\right) \cdot\left(\Theta_{l_{1}, l_{2}} h(m(\chi), \underline{\underline{r}}, \underline{\kappa})-h(m(\chi), \underline{\underline{r}}, \underline{\kappa})\right)+\left(\widehat{\beta}^{\chi}-\beta\left(\kappa_{l_{2}}\right)\right) \cdot h(m(\chi), \underline{\underline{r}}, \underline{\kappa})\right\} \\
& =\int \nu^{\chi}(\mathrm{d}(\underline{\underline{r}}, \underline{\kappa}))\left\{\beta\left(\kappa_{l_{1}}\right) \cdot \Theta_{l_{1}, l_{2}} h(m(\chi), \underline{\underline{r}}, \underline{\kappa})-\beta\left(\kappa_{l_{2}}\right) \cdot h(m(\chi), \underline{\underline{r}}, \underline{\kappa})\right\} \\
& -\widehat{\beta}^{\chi} \int \nu^{\chi}(\mathrm{d}(\underline{\underline{r}}, \underline{\kappa})) h(m(\chi), \underline{\underline{r}}, \underline{\kappa})+\widehat{\beta}^{\chi} \int \nu^{\chi}(\mathrm{d}(\underline{\underline{r}}, \underline{\kappa})) h(m(\chi), \underline{\underline{r}}, \underline{\kappa}) \\
& =0 .
\end{aligned}
$$

On the other hand, assume that $l_{2} \in \mathbb{N}$ is such that the function $h$ does not depend on $r_{i \wedge l_{2}, i \vee l_{2}}$ for all $i \in \mathbb{N}$ and also does not depend on $\kappa_{l_{2}}$. Then once more

$$
\begin{aligned}
& \int \nu^{\chi}(\mathrm{d}(\underline{\underline{r}}, \underline{\kappa}))\left\{\beta\left(\kappa_{l_{1}}\right) \cdot\left(\Theta_{l_{1}, l_{2}} h(m(\chi), \underline{\underline{r}}, \underline{\kappa})-h(m(\chi), \underline{\underline{r}}, \underline{\kappa})\right)+\left(\widehat{\beta}^{\chi}-\beta\left(\kappa_{l_{2}}\right)\right) \cdot h(m(\chi), \underline{\underline{r}}, \underline{\kappa})\right\} \\
& =\int \nu^{\chi}(\mathrm{d}(\underline{\underline{r}}, \underline{\kappa})) \beta\left(\kappa_{l_{1}}\right) \cdot(h(m(\chi), \underline{\underline{r}}, \underline{\kappa})-h(m(\chi), \underline{\underline{r}}, \underline{\kappa})) \\
& +\widehat{\beta}^{\chi} \int \nu^{\chi}(\mathrm{d}(\underline{\underline{r}}, \underline{\kappa})) h(m(\chi), \underline{\underline{r}}, \underline{\kappa})-\widehat{\beta}^{\chi} \int \nu^{\chi}(\mathrm{d}(\underline{\underline{r}}, \underline{\kappa})) h(m(\chi), \underline{\underline{r}}, \underline{\kappa}) \\
& =0 .
\end{aligned}
$$

This shows that indeed $\Omega_{\text {resample }}^{\beta} F^{h}$ reduces to a sum with finitely many summands and is thus well-defined whenever $h$ has finite degree.

We are now in a position to state our second main result.

Theorem 2 (The martingale problem). Let for each $N \in \mathbb{N}, \mathcal{X}^{N}$ be the tree-valued $\left(\ell_{N}, \zeta_{N}, \alpha_{N}\right)$ trait-dependent branching dynamics with mutation and competition such that the assumptions of Theorem 1 hold. If $\mathcal{X}_{0}^{N}$ converges weakly to $\mathcal{X}_{0}$ in $\mathbb{M}^{K}$ with $\mathbb{E}\left[\left(m\left(\mathcal{X}_{0}\right)\right)^{3}\right]<\infty$, then any limit process $\mathcal{X}$ satisfies the $(\Omega, \mathcal{D}(\Omega))$-martingale problem.

Remark 2.13 (Relation with other tree-valued dynamics). Several related models of evolving genealogies have been considered so far in the literature.

(1) Tree-valued Fleming-Viot with mutation and selection. In DGP12] the authors consider the evolving genealogies of a trait-dependent individual based particle system with mutation and selection, where the total mass is fixed, say $m\left(\chi_{t}\right) \equiv 1$ (and thus $\left.\Omega_{\text {total mass }}^{\beta, \Gamma} \equiv 0\right)$, and the natural branching rate does not depend on the trait, that is, $\beta \equiv \widehat{\beta}$ (and thus $\Omega_{\beta \text {-reweigh }}^{\beta} \equiv 0$ ), and their single mutation is a continuous state and continuous time Markov chain on $K$, that is, of the form

$$
A f(\kappa):=\vartheta \cdot \int_{K}\left(a\left(\kappa, \mathrm{d} \kappa^{\prime}\right)-\delta_{\kappa}\left(\mathrm{d} \kappa^{\prime}\right)\right) f\left(\kappa^{\prime}\right)
$$

(which is the limit of rare mutations). Moreover, their genealogical distances grow with time no matter whether mutation occurs or not (might be interpreted as $p=1$ ). In this 
particular case, $\Gamma\left(m, r_{1,2}, \kappa_{1}, \kappa_{2}\right) \equiv \tilde{\Gamma}\left(r_{1,2}, \kappa_{1}, \kappa_{2}\right)$, for some bounded $\tilde{\Gamma}: \mathbb{R}_{+} \times K \times K$, and (2.37) reduces to $\Omega=\Omega_{\text {trait mutation }}^{\widehat{\beta}, 1, \frac{1}{\beta} A}+\Omega_{\text {growth }}^{1,1}+\Omega_{\Gamma \text {-reweigh }}^{\tilde{\Gamma}}+\Omega_{\text {resample }}^{\widehat{\beta}}$.

(2) Tree-valued (neutral) Fleming-Viot (without mutation). In the particular case without selection and mutation, that is, where $\Gamma \equiv 0$ (and thus $\Omega_{\Gamma \text {-reweigh }}^{\Gamma} \equiv 0$ ) and $A \equiv \operatorname{Id}$ (and thus $\Omega_{\text {trait mutation }}^{p, \beta, A} \equiv 0$ ), this reduces to $\Omega=\Omega_{\text {growth }}^{1,1}+\Omega_{\text {resample }}^{\widehat{\beta}}$, which is the operator corresponding to the tree-valued Fleming-Viot diffusion constructed in GPW13.

(3) Historical processes with mutation and selection. In MT12 consider a traitdependent individual based branching model with mutation and selection is considered, where the selection takes the trait history of an individual into account. If we restrict their model to the case, where the dependence on the historical trait path is only through the current trait, this results in a measure-valued process (and thus $\Omega_{\text {growth }}^{p, \beta} \equiv 0$ ) with the particular set-up

$$
\Gamma\left(m, r_{1,2}, \kappa_{1}, \kappa_{2}\right)=b\left(\kappa_{2}\right)-d\left(\kappa_{2}\right)-m \cdot U\left(\kappa_{1}, \kappa_{2}\right)
$$

and

$$
A f(\kappa):=\frac{\sigma^{2}}{2} f^{\prime \prime}(\kappa)
$$

Thus (2.37) reads as follows: $\Omega:=\Omega_{\text {total mass }}^{\beta, \Gamma}+\Omega_{\text {trait mutation }}^{p, \beta, A}+\Omega_{\Gamma \text {-reweigh }}^{\Gamma}+\Omega_{\text {natural branching, }}^{\beta}$, where the generator acts for instance on functions $F^{h}$ of the particular form

$$
h\left(m,\left(r_{i, j}\right)_{1 \leq i<j \leq n},\left(\kappa_{i}\right)_{1 \leq i \leq n}\right):=m^{n} \cdot f(\underline{\kappa}) .
$$

The latter model has been extended in [Kli14] by considering general mutation operators on Polish trait spaces.

\section{UNIFORM MOMENT BOUNDS FOR THE DISCRETE MODELS}

Throughout this section we assume that Assumptions 1, 2 and 3 hold. Recall the treevalued $(\ell, \zeta, \alpha)$-trait-dependent branching dynamics with mutation and competition, $\mathcal{X}^{(\ell, \zeta, \alpha)}$, as defined in Subsection 2.2 (Definition 2.8) by means of the natural branching rate $\beta: K \rightarrow \mathbb{R}_{+}$, the competition birth rate $\gamma^{\text {death }}: \mathbb{R}_{+} \times \mathbb{R}_{+} \times K^{2} \rightarrow \mathbb{R}_{+}$, the competition death rate $\gamma^{\text {death }}$ : $\mathbb{R}_{+} \times \mathbb{R}_{+} \times K^{2} \rightarrow \mathbb{R}_{+}$, and the mutation kernel $\alpha$. Its generator $\Omega^{(\ell, \zeta, \alpha)}$ acts on bounded functions 
$F$ on $\mathbb{M}^{K,(\ell, \zeta)}$ as follows (cf. (2.17)-(2.23)):

$(3.1)$

$$
\begin{aligned}
& \Omega^{(\ell, \zeta, \alpha)} F(\chi) \\
& =:\left(\Omega_{\text {death }}^{(\ell, \zeta, \alpha)}+\Omega_{\text {birth }}^{(\ell, \zeta, \alpha)}\right) F(\chi) \\
& =\frac{m(\chi)}{\zeta} \int_{(X \times K)^{2}} \bar{\mu}^{\otimes 2}\left(\mathrm{~d}\left(\left(x_{1}, \kappa_{1}\right),\left(x_{2}, \kappa_{2}\right)\right)\right)\left\{\frac{\beta\left(\kappa_{2}\right)}{\zeta}+\gamma^{\text {death }}\left(m(\chi), r\left(x_{1}, x_{2}\right), \kappa_{1}, \kappa_{2}\right)\right\} \\
& \quad \cdot\left(F\left(\left(X, r, \mu-\zeta \cdot \delta_{\left(x_{2}, \kappa_{2}\right)}\right)\right)-F(\chi)\right) \\
& +\frac{m(x)}{\zeta} \int_{(X \times K)^{2}} \bar{\mu}^{\otimes 2}\left(\mathrm{~d}\left(\left(x_{1}, \kappa_{1}\right),\left(x_{2}, \kappa_{2}\right)\right)\right)\left\{\frac{\beta\left(\kappa_{2}\right)}{\zeta}+\gamma^{\operatorname{birth}}\left(m(\chi), r\left(x_{1}, x_{2}\right), \kappa_{1}, \kappa_{2}\right)\right\} \int_{K} \widehat{\alpha}\left(\kappa_{2}, \mathrm{~d} \tilde{\kappa}_{2}\right) \\
& \quad \cdot\left(F\left(\left(X \uplus\{z\}, r^{\left(x_{2}, z\right), \ell}, \mu+\zeta \cdot \delta_{\left(z, \tilde{\kappa}_{2}\right)}\right)\right)-F(\chi)\right) .
\end{aligned}
$$

In the proof of tightness we will make use of the following uniform moment bounds.

Proposition 3.1 (Uniform moment bounds). Let $\mathcal{X}:=\left(\mathcal{X}^{(\ell, \zeta, \alpha)}\right)_{t \in[0, \infty)}$ be the $(\ell, \zeta, \alpha)$-traitdependent branching dynamics with mutation and competition. Fix $q \in \mathbb{N}$.

(i) Then for all $(\ell, \zeta, \alpha)$ with $\zeta \leq 1$, and all $t>0$,

$$
\sup _{s \in[0, t]} \mathbb{E}\left[\left(m\left(\mathcal{X}_{s}^{(\ell, \zeta, \alpha)}\right)\right)^{q}\right] \leq\left(1+\mathbb{E}\left[\left(m\left(\mathcal{X}_{0}^{(\ell, \zeta, \alpha)}\right)\right)^{q}\right]\right) \cdot e^{C_{q} \cdot\left(2 \bar{\beta}+\bar{\gamma}_{b}\right) \cdot t}
$$

for some $C_{q}>0$.

(ii) Assume that in addition Assumption 6 holds. If the initial masses satisfy

$$
\sup _{\zeta \in(0,1]} \mathbb{E}\left[\left(m\left(\mathcal{X}_{0}^{(\ell, \zeta, \alpha)}\right)\right)^{2 q+1}\right]<\infty,
$$

then for all $t>0$, there is a constant $C_{q, \bar{\beta}, \bar{\gamma}_{b}, \bar{\gamma}_{d}, t} \in(0, \infty)$ such that for all $(\ell, \zeta, \alpha)$ with $\zeta \leq 1$

$$
\mathbb{E}\left[\sup _{s \in[0, t]}\left(m\left(\mathcal{X}_{s}^{(\ell, \zeta, \alpha)}\right)\right)^{q}\right] \leq C_{q, \bar{\beta}, \bar{\gamma}_{b}, \bar{\gamma}_{d}, t} .
$$

(iii) Let $q=1$ and $\gamma^{\text {death }}\left(m, r, \kappa, \kappa^{\prime}\right) \equiv 0$, that is $\bar{\gamma}_{d}=0$. Under the assumptions of (ii), including (3.3), for all $t>0$ and $\delta, \epsilon>0$ there exists $m_{0}>0$ small enough such that for all $(\ell, \zeta, \alpha)$ with $\zeta \leq 1$,

$$
\mathbb{P}\left(\sup _{s \in[0, t]} m\left(\mathcal{X}_{s}^{(\ell, \zeta, \alpha)}\right) \geq \delta\right) \leq \epsilon
$$

if $\mathbb{E}\left[m\left(\mathcal{X}_{0}^{(\ell, \zeta, \alpha)}\right] \leq m_{0}\right.$.

Proof. The proof is inspired by [FM04, Step 2 of the proof of Theorem 5.6]. Fix $q \geq 1$, and put for $L \geq 0$,

$$
F^{q, L}(\chi):=(m(x) \wedge L)^{q} .
$$


Then $F^{q, L} \in \mathcal{D}\left(\Omega^{(\ell, \zeta, \alpha)}\right)$, and

$$
\begin{aligned}
& \Omega^{(\ell, \zeta, \alpha)} F^{q, L}(\chi) \\
& =\zeta^{-1} \cdot m(\chi) \cdot\left(\zeta^{-1} \cdot \widehat{\beta}^{\chi}+\widehat{\gamma}^{\operatorname{death}}(m(\chi))\right) \cdot\left(((m(\chi)-\zeta) \wedge L)^{q}-(m(\chi) \wedge L)^{q}\right) \\
& +\zeta^{-1} \cdot m(\chi) \cdot\left(\zeta^{-1} \cdot \widehat{\beta}^{\chi}+\widehat{\gamma}^{\operatorname{birth}}(m(\chi))\right) \cdot\left(((m(\chi)+\zeta) \wedge L)^{q}-(m(\chi) \wedge L)^{q}\right) .
\end{aligned}
$$

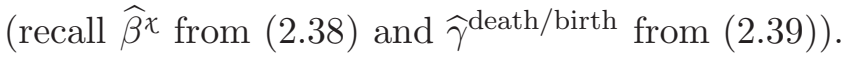

Thus, for all $t \geq 0$,

$$
F^{q, L}\left(\mathcal{X}_{t}^{(\ell, \zeta, \alpha)}\right)=F^{q, L}\left(\mathcal{X}_{0}^{(\ell, \zeta, \alpha)}\right)+\int_{0}^{t} \mathrm{~d} s \Omega^{(\ell, \zeta, \alpha)} F^{q, L}\left(\mathcal{X}_{s}^{(\ell, \zeta, \alpha)}\right)+M_{t}^{q, L},
$$

where $\left(M_{t \wedge \tau_{M}}^{q, L}\right)_{t \geq 0}$ with

$$
\tau_{M}:=\inf \left\{t \geq 0: m\left(\mathcal{X}_{t}^{(\ell, \zeta, \alpha)}\right) \geq M\right\}, M>0
$$

is a càdlàg $L^{2}$-martingale starting from 0 and with quadratic variation

$$
\langle M\rangle_{t \wedge \tau_{M}}=\int_{0}^{t \wedge \tau_{M}} \mathrm{~d} s\left(\Omega^{(\ell, \zeta, \alpha)}\left(F^{2}\right)-2 F \Omega^{(\ell, \zeta, \alpha)} F\right)\left(\mathcal{X}_{s}^{(\ell, \zeta, \alpha)}\right) .
$$

(i). To obtain an upper bound for $F^{q, L}\left(\mathcal{X}_{t \wedge \tau_{M}}^{(\ell, \zeta, \alpha)}\right)$, we can drop the competition death term in (3.7) respectively (3.8), and use that with $C_{q}:=2^{q}-1$ for all $m>0$ and $\zeta \in(0,1]$ (note that the case $q=1$ is trivial),

$$
\begin{aligned}
m \cdot\left|(m+\zeta)^{q}-2 m^{q}+(m-\zeta)^{q}\right| & =2 m \zeta^{2} \cdot\left|\sum_{k=0, q-k \text { even }}^{q-2}\left(\begin{array}{l}
q \\
k
\end{array}\right) m^{k} \zeta^{q-k-2}\right| \\
& \leq 2 C_{q} \cdot \zeta^{2} \cdot\left(1+m^{q}\right)
\end{aligned}
$$

as well as

$$
m \cdot\left|(m+\zeta)^{q}-m^{q}\right| \leq C_{q} \cdot \zeta \cdot\left(1+m^{q}\right) .
$$

Therefore by Assumptions 1 and 2, for all $M>0$ and all $L \geq M+\zeta$,

$$
\begin{aligned}
& F^{q, L}\left(\mathcal{X}_{t \wedge \tau_{M}}^{(\ell, \zeta, \alpha)}\right) \\
& \leq F^{q, L}\left(\mathcal{X}_{0}^{(\ell, \zeta, \alpha)}\right)+M_{t \wedge \tau_{M}}^{q, L} \\
& +\zeta^{-2} \cdot \int_{0}^{t \wedge \tau_{M}} \mathrm{~d} s m\left(\mathcal{X}_{s}^{(\ell, \zeta, \alpha)}\right) \cdot \widehat{\beta}^{\mathcal{X}_{s}^{(\ell, \zeta, \alpha)}} \cdot\left(\left(m\left(\mathcal{X}_{s}^{(\ell, \zeta, \alpha)}\right)-\zeta\right)^{q}+\left(m\left(\mathcal{X}_{s}^{(\ell, \zeta, \alpha)}\right)+\zeta\right)^{q}-2\left(m\left(\mathcal{X}_{s}^{(\ell, \zeta, \alpha)}\right)\right)^{q}\right) \\
& \left.+\zeta^{-1} \cdot \int_{0}^{t \wedge \tau_{M}} \mathrm{~d} s m\left(\mathcal{X}_{s}^{(\ell, \zeta, \alpha)}\right) \cdot \widehat{\gamma}^{\text {birth }}\left(m\left(\mathcal{X}_{s}^{(\ell, \zeta, \alpha)}\right)\right) \cdot\left(\left(m\left(\mathcal{X}_{s}^{(\ell, \zeta, \alpha)}\right)+\zeta\right)\right)^{q}-\left(m\left(\mathcal{X}_{s}^{(\ell, \zeta, \alpha)}\right)\right)^{q}\right) \\
& \leq F^{q, L}\left(\mathcal{X}_{0}^{(\ell, \zeta, \alpha)}\right)+M_{t \wedge \tau_{M}}^{q, L} \\
& +C_{q} \cdot \int_{0}^{t \wedge \tau_{M}} \mathrm{~d} s\left\{2 \bar{\beta} \cdot\left(\left(m\left(\mathcal{X}_{s}^{(\ell, \zeta, \alpha)}\right)\right)^{q}+1\right)+\bar{\gamma}_{b} \cdot\left(\left(m\left(\mathcal{X}_{s}^{(\ell, \zeta, \alpha)}\right)\right)^{q}+1\right)\right\} \cdot
\end{aligned}
$$


Hence by monotone convergence as $L \uparrow \infty$, for all $M$,

$$
\begin{aligned}
& \mathbb{E}\left[\left(m\left(\mathcal{X}_{t \wedge \tau_{M}}^{(\ell, \zeta, \alpha)}\right)\right)^{q}\right] \\
& \leq \mathbb{E}\left[\left(m\left(\mathcal{X}_{0}^{(\ell, \zeta, \alpha)}\right)\right)^{q}\right]+C_{q} \cdot\left(2 \bar{\beta}+\bar{\gamma}_{b}\right) \cdot \int_{0}^{t} \mathrm{~d} s\left\{\mathbb{E}\left[\left(m\left(\mathcal{X}_{s \wedge \tau_{M}}^{(\ell, \zeta, \alpha)}\right)\right)^{q}\right]+1\right\} .
\end{aligned}
$$

We therefore obtain (3.2) from Gronwall's lemma and subsequently taking $M \rightarrow \infty$.

(ii). Now we move the supremum over time inside the expectation. Using once more (3.13), for all $L \geq M+\zeta$,

$$
\begin{aligned}
\sup _{s \in\left[0, t \wedge \tau_{M}\right]} F^{q, L}\left(\mathcal{X}_{s}^{(\ell, \zeta, \alpha)}\right) \leq & F^{q, L}\left(\mathcal{X}_{0}\right)+\sup _{s \in\left[0, t \wedge \tau_{M}\right]}\left|M_{s}^{q, L}\right| \\
& +C_{q} \cdot\left(2 \bar{\beta}+\bar{\gamma}_{b}\right) \cdot \int_{0}^{t \wedge \tau_{M}} \mathrm{~d} s\left(\left(m\left(\mathcal{X}_{s}^{(\ell, \zeta, \alpha)}\right)\right)^{q}+1\right) .
\end{aligned}
$$

Taking expectations,

$$
\begin{aligned}
& \mathbb{E}\left[\sup _{s \in\left[0, t \wedge \tau_{M}\right]}\left(m\left(\mathcal{X}_{s}^{(\ell, \zeta, \alpha)}\right)\right)^{q}\right] \\
& \leq \mathbb{E}\left[\left(m\left(\mathcal{X}_{0}^{(\ell, \zeta, \alpha)}\right)\right)^{q}\right]+\mathbb{E}\left[\sup _{s \in\left[0, t \wedge \tau_{M}\right]}\left|M_{s}^{q, L}\right|\right]+C_{q} \cdot\left(2 \bar{\beta}+\bar{\gamma}_{b}\right) \cdot \int_{0}^{t} \mathrm{~d} s \mathbb{E}\left[\left(\left(m\left(\mathcal{X}_{s}^{(\ell, \zeta, \alpha)}\right)\right)^{q}+1\right) \cdot 1\left\{s \leq \tau_{M}\right\}\right] \\
& \leq \mathbb{E}\left[\left(m\left(\mathcal{X}_{0}^{(\ell, \zeta, \alpha)}\right)\right)^{q}\right]+\mathbb{E}\left[\sup _{s \in\left[0, t \wedge \tau_{M}\right]}\left|M_{s}^{q, L}\right|\right]+C_{q} \cdot\left(2 \bar{\beta}+\bar{\gamma}_{b}\right) \cdot \int_{0}^{t} \mathrm{~d} s \mathbb{E}\left[\left(\left(m\left(\mathcal{X}_{s \wedge \tau_{M}}^{(\ell, \zeta, \alpha)}\right)\right)^{q}+1\right)\right] .
\end{aligned}
$$

By a Burkholder-Davis-Gundy inequality and Cauchy-Schwarz's inequality, there is a $C<\infty$ such that

$$
\mathbb{E}\left[\sup _{s \in\left[0, t \wedge \tau_{M}\right]}\left|M_{s}^{q, L}\right|\right] \leq C \cdot \mathbb{E}\left[\left\langle M_{\cdot}^{q, L}\right\rangle_{t \wedge \tau_{M}}^{\frac{1}{2}}\right] \leq C \cdot\left(\mathbb{E}\left[\left\langle M_{\cdot}^{q, L}\right\rangle_{t \wedge \tau_{M}}\right]\right)^{\frac{1}{2}} .
$$

We conclude with (3.10) that for all $L \geq M+\zeta$, $(3.18)$

$$
\begin{aligned}
& \mathbb{E}\left[\left\langle M_{\cdot}^{q, L}\right\rangle_{\left.t \wedge \tau_{M}\right]}\right. \\
& \leq \int_{0}^{t} \mathrm{~d} s \mathbb{E}\left[\frac{m\left(\mathcal{X}_{s}^{(\ell, \zeta, \alpha)}\right)}{\zeta}\left(\left(m\left(\mathcal{X}_{s}^{(\ell, \zeta, \alpha)}\right)-\zeta\right)^{q}+\left(m\left(\mathcal{X}_{s}^{(\ell, \zeta, \alpha)}\right)\right)^{q}\right)^{2} \cdot\left(\frac{\widehat{\beta}^{\mathcal{X}_{s}^{(\ell, \zeta, \alpha)}}}{\zeta}+\widehat{\gamma}^{\text {death }}\left(m\left(\mathcal{X}_{s}^{(\ell, \zeta, \alpha)}\right)\right)\right) ; s \leq \tau_{M}\right] \\
& +\int_{0}^{t} \mathrm{~d} s \mathbb{E}\left[\frac{m\left(\mathcal{X}_{s}^{(\ell, \zeta, \alpha)}\right)}{\zeta}\left(\left(m\left(\mathcal{X}_{s}^{(\ell, \zeta, \alpha)}\right)+\zeta\right)^{q}+\left(m\left(\mathcal{X}_{s}^{(\ell, \zeta, \alpha)}\right)\right)^{q}\right)^{2} \cdot\left(\frac{\widehat{\beta}^{(\ell, \zeta, \alpha)}}{\zeta}+\widehat{\gamma}^{\operatorname{birth}}\left(m\left(\mathcal{X}_{s}^{(\ell, \zeta, \alpha)}\right)\right)\right) ; s \leq \tau_{M}\right] \\
& \leq C \cdot\left(2 \bar{\beta}+\bar{\gamma}_{b}+\bar{\gamma}_{d}\right) \cdot \int_{0}^{t} \mathrm{~d} s\left(1+\mathbb{E}\left[\left(m\left(\mathcal{X}_{s}^{(\ell, \zeta, \alpha)}\right)\right)^{2 q+1}\right]\right),
\end{aligned}
$$

which gives the claim after combining (3.2), (3.16), (3.17) and (3.18).

(iii). By (ii) we already know that the integrals in question are well-defined. For $q=1$, instead of (3.11) and (3.12), use that

$$
m \cdot|(m+\zeta)-2 m+(m-\zeta)|=0 \text { and } m \cdot|(m+\zeta)-m|=m \zeta
$$


to conclude as in (3.13) that

$$
m\left(\mathcal{X}_{t \wedge \tau_{M}}^{(\ell, \zeta, \alpha)}\right) \wedge L=m\left(\mathcal{X}_{0}^{(\ell, \zeta, \alpha)}\right) \wedge L+M_{t \wedge \tau_{M}}^{1, L}+\int_{0}^{t \wedge \tau_{M}} \mathrm{~d} s m\left(\mathcal{X}_{s}^{(\ell, \zeta, \alpha)}\right) \cdot \widehat{\gamma}^{\operatorname{birth}}\left(m\left(\mathcal{X}_{s}^{(\ell, \zeta, \alpha)}\right)\right) .
$$

Hence, $m\left(\mathcal{X}_{t \wedge \tau_{M}}^{(\ell,, \alpha)}\right) \wedge L$ is a non-negative submartingale and Gronwall's lemma yields $\mathbb{E}\left[m\left(\mathcal{X}_{t}^{(\ell, \zeta, \alpha)}\right)\right] \leq$ $\mathbb{E}\left[m\left(\mathcal{X}_{0}^{(\ell, \zeta, \alpha)}\right)\right] \cdot e^{\bar{\gamma}_{b} t}$. In combination with Doob's inquality we obtain for all $\delta>0$,

$$
\mathbb{P}\left(\sup _{s \in[0, t]} m\left(\mathcal{X}_{s}^{(\ell, \zeta, \alpha)}\right) \geq \delta\right) \leq \delta^{-2} \mathbb{E}\left[\left(m\left(\mathcal{X}_{t}^{(\ell, \zeta, \alpha)}\right)\right)^{2}\right] .
$$

We use (3.10) again, together with $m\left((m \pm \zeta)^{2}-m^{2}-2 m((m \pm \zeta)-m)\right)=m \zeta^{2}$, to get

$$
\mathbb{E}\left[\left\langle M_{\cdot}^{q, 1}\right\rangle_{t \wedge \tau_{M}}\right] \leq\left(2 \bar{\beta}+\bar{\gamma}_{b}\right) \cdot \int_{0}^{t} \mathrm{~d} s \mathbb{E}\left[m\left(\mathcal{X}_{s}^{(\ell, \zeta, \alpha)}\right)\right] \leq\left(2 \bar{\beta}+\bar{\gamma}_{b}\right) \cdot m_{0} \cdot \int_{0}^{t} \mathrm{~d} s e^{\bar{\gamma}_{b} s} .
$$

Now apply Itô's formula to see that

$$
\mathbb{E}\left[\left(m\left(\mathcal{X}_{t}^{(\ell, \zeta, \alpha)}\right)\right)^{2}\right] \leq m_{0}^{2}+2 \bar{\gamma}_{b} \cdot \int_{0}^{t} \mathrm{~d} s \mathbb{E}\left[\left(m\left(\mathcal{X}_{t}^{(\ell, \zeta, \alpha)}\right)\right)^{2}\right]+\left(2 \bar{\beta}+\bar{\gamma}_{b}\right) \cdot m_{0} \cdot \int_{0}^{t} \mathrm{~d} s e^{\bar{\gamma}_{b} s} .
$$

Another application of Gronwall's lemma yields

$$
\mathbb{E}\left[\left(m\left(\mathcal{X}_{t}^{(\ell, \zeta, \alpha)}\right)\right)^{2}\right] \leq m_{0} \cdot\left(m_{0}+\left(2 \bar{\beta}+\bar{\gamma}_{b}\right) \cdot \int_{0}^{t} \mathrm{~d} s e^{\bar{\gamma}_{b} s}\right) \cdot e^{2 \bar{\gamma}_{b} t} .
$$

Use $m_{0}$ small enough in (3.21) to conclude the claim.

\section{UNIFORM CONVERGENCE OF GENERATORS}

Recall the $(\ell, \zeta, \alpha)$-trait-dependent branching with mutation and competition from Definition 2.8, its state space $\mathbb{M}^{K,(\ell, \zeta)}$ from (2.12), and its generator $\Omega^{(\ell, \zeta, \alpha)}=\Omega_{\text {birth }}^{(\ell, \zeta, \alpha)}+\Omega_{\text {death }}^{(\ell, \zeta, \alpha)}$ acting on bounded measurable functions from (3.1). In the following we abbreviate

$$
\Omega_{N}:=\Omega^{\left(\frac{1}{N}, \frac{1}{N}, \alpha_{N}\right)}
$$

and

$$
\mathbb{M}_{N}^{K}:=\mathbb{M}^{K,\left(\frac{1}{N}, \frac{1}{N}\right)}
$$

with a family $\left\{\alpha_{N} ; N \in \mathbb{N}\right\}$ of mutation operators satisfying Assumption 4 . Furthermore, recall the tree-valued trait-dependent branching with mutation and competition from Definition 2.10, its state space $\mathbb{M}^{K}$ from (2.4), and its generator $(\Omega, \mathcal{D}(\Omega)$ ) from (2.36) and (2.37).

As a first step in proving tightness we are proving the uniform convergence of the generators in this section. Put

$$
\begin{aligned}
\widetilde{\Pi}:=\{ & F^{h} \in \Pi^{2,1, A} \cap \Pi_{\text {finite }} \text { of the form } h(m, \underline{\underline{r}}, \underline{\kappa})=g(m) \cdot \phi\left(\left(r_{i, j}\right)_{1 \leq i<j \leq n}\right) \cdot f\left(\left(\kappa_{i}\right)_{1 \leq i \leq n}\right) \text { with } \\
& n \in \mathbb{N}_{0}, g \in \mathcal{C}_{b}^{3}\left(\mathbb{R}_{+}\right) \text {such that } g^{\prime}(0)=0, \limsup _{m \rightarrow \infty} m \tilde{\gamma}(m)\left|g^{\prime}(m)\right|<\infty, \\
& \limsup _{m \rightarrow \infty}\left|g^{\prime \prime}(m)\right|<\infty, \limsup _{m \rightarrow \infty}(1 \vee \tilde{\gamma}(m))|g(m)|<\infty \text { and } \\
& \left.\lim _{\epsilon \downarrow 0} \limsup _{m \rightarrow \infty} m \cdot(1 \vee \tilde{\gamma}(m)) \max _{\xi \in[m-\epsilon, m+\epsilon]}\left|g^{\prime \prime \prime}(\xi)\right|<\infty\right\},
\end{aligned}
$$


where $\tilde{\gamma}$ is as in Assumption 3 ,

Definition 4.1 (Degree of $h$ and degree of $F$ ). Let $h \in \mathcal{C}_{b}\left(\mathbb{R}_{+} \times \mathbb{R}_{+}^{\left(\begin{array}{c}\mathbb{N} \\ 2\end{array}\right)} \times K^{\mathbb{N}}\right)$ depend on $\left(m,\left(r_{i, j}\right)_{1 \leq i<j},\left(\kappa_{i}\right)_{i \in \mathbb{N}}\right)$ only through $\left(m,\left(r_{i, j}\right)_{1 \leq i<j: i, j \in I},\left(\kappa_{i}\right)_{i \in I}\right)$ for a finite set $I \subseteq \mathbb{N}$. The smallest number $\# I \in \mathbb{N}$ with this property is referred to as the degree of $h$. For a polynomial $F: \mathbb{M}^{K} \rightarrow \mathbb{R}$ of finite degree we denote by $\operatorname{deg}(F)$ the smallest number for which $F$ is of the form (2.32) for a function $h \in \mathcal{C}_{b}\left(\mathbb{R}_{+} \times \mathbb{R}_{+}^{\left(\begin{array}{c}\mathbb{N} \\ 2\end{array}\right)} \times K^{\mathbb{N}}\right)$ of degree $\operatorname{deg}(h)$.

Notation 4.2. For $h \in \tilde{\Pi}$ with $h(\cdot, \underline{\underline{r}}, \underline{\kappa})=g(m) \phi(\underline{\underline{r}}) f(\underline{\kappa})$ and $n:=\operatorname{deg}(h)$ write $F^{g,(n, \phi, f)}:=F^{h}$.

The main result reads as follows:

Proposition 4.3 (Convergence of generators). For all $F \in \tilde{\Pi}$, under Assumptions 1, 2, 3 and 4

$$
\lim _{N \rightarrow \infty} \sup _{\chi \in \mathbb{M}_{N}^{K}}\left|\Omega_{N} F(\chi)-\Omega F(\chi)\right|=0 .
$$

Throughout this section we will fix a sequence $\left(a_{N}\right)_{N \in \mathbb{N}}, a_{N} \rightarrow \infty$ for $N \rightarrow \infty$ but slow enough such that

$$
\frac{a_{N}}{N} \underset{N \rightarrow \infty}{\longrightarrow} 0
$$

To prepare the proof of Proposition 4.3 we first show the following lemma.

Lemma 4.4. If $F \in \widetilde{\Pi}$, then

$$
\sup _{\chi: m(\chi) \leq \frac{a_{N}}{N}}|\Omega F(x)| \underset{N \rightarrow \infty}{\longrightarrow} 0
$$

Proof. Recall that $\Omega F(\chi)=0$ if $m(\chi)=0$ or if $F$ is constant. Fix therefore $\chi \in \mathbb{M}^{K}$ with $0<m(\chi) \leq \frac{\alpha_{N}}{N}$. As $\Omega$ is linear, we may assume without loss of generality that $g(0)=0$. Then recalling (2.37),

$|\Omega F(\chi)|$

$$
\begin{aligned}
& \leq\left|\Omega_{\text {total mass }}^{\beta, \Gamma} F(\chi)\right|+\left|\Omega_{\text {trait mutation }}^{p, \beta, A} F(\chi)\right|+\left|\Omega_{\text {growth }}^{p, \beta} F(\chi)\right|+\left|\Omega_{\Gamma-\text { reweigh }}^{\Gamma} F(\chi)\right|+\left|\Omega_{\text {natural branching }}^{\beta} F(\chi)\right| \\
& \leq\|\phi f\| \cdot \sup _{m \in\left[0, \frac{a_{N}}{N}\right]}\left(\widehat{\Gamma}(m) m\left|g^{\prime}(m)\right|+\bar{\beta} m\left|g^{\prime \prime}(m)\right|+2 \operatorname{deg}(h) \bar{\beta}\left|g^{\prime}(m)\right|\right) \\
& +p\|\phi\| \cdot \bar{\beta} \sum_{l=1}^{\operatorname{deg}(h)}\left\|A^{(l)} f\right\| \cdot \sup _{m \in\left[0, \frac{a_{N}}{N}\right]}|g(m)|+2 p \bar{\beta}\|f\| \cdot \sum_{1 \leq l_{1}<l_{2} \leq \operatorname{deg}(h)}\left\|\frac{\partial \phi}{\partial r_{l_{1}, l_{2}}}\right\| \cdot \sup _{m \in\left[0, \frac{a_{N}}{N}\right]}|g(m)| \\
& +2 \operatorname{deg}(h) \cdot\|\phi f\| \cdot \sup _{m \in\left[0, \frac{a_{N}}{N}\right]}\left(\tilde{\gamma}(m)+\bar{\gamma}_{b}\right)|g(m)| \\
& +2\|\phi f\| \bar{\beta}\left(\operatorname{deg}(h)+2(\operatorname{deg}(h))^{2}\right) \cdot \sup _{m \in\left[0, \frac{a_{N}}{N}\right]} \frac{|g(m)|}{m} .
\end{aligned}
$$

Thus (4.6) follows as $g$ is continuously differentiable with $g^{\prime}(0)=0$ and $\tilde{\gamma}$ is continuous. 
We continue with the proof of Proposition 4.3 and turn to the approximating operators.

Proof of Proposition 4.3. Fix $F=F^{g,(n, \phi, f)} \in \tilde{\Pi}, N \in \mathbb{N}$, and $\chi=\overline{(X, r, \mu)} \in \mathbb{M}_{N}^{K}$. We assume once more without loss of generality that $g(0)=0$.

Notice that if $m(\chi)=0$, then $\Omega_{N} F(\chi)=0$ for all $N \in \mathbb{N}$ by definition. Also, if $m(\chi)=0$, then $\Omega F(x)=0$, cf. below (2.37).

If $\chi$ is such that $m(\chi)=\frac{1}{N}$, that is, $\mu=\frac{1}{N} \delta_{(x, k)}$ say, then

$$
\begin{aligned}
& \left|\Omega_{N} F(\chi)\right| \\
& \leq\left|\Omega_{N}^{\text {death }} F(\chi)\right|+\left|\Omega_{N}^{\text {birth }} F(\chi)\right| \\
& \leq \\
& \left.\quad+N \cdot \beta(k)+\gamma_{\text {death }}\left(\frac{1}{N}, 0, k, k\right)\right)\left|g\left(\frac{1}{N}\right) \phi(\underline{\underline{0}}) f(\underline{k})\right| \\
& \quad+\left(N \cdot \beta(k)+\gamma_{\text {birth }}\left(\frac{1}{N}, 0, k, k\right)\right) \\
& \quad \cdot\left|\int \widehat{\alpha}_{N}(k, \mathrm{~d} \tilde{k})\left\{g\left(\frac{2}{N}\right) \int\left(\frac{1}{2} \delta_{(x, k)}+\frac{1}{2} \delta_{(z, \tilde{k})}\right)\left(\mathrm{d}\left(\underline{x}^{\prime}, \underline{k}^{\prime}\right)\right) \phi\left(\underline{\underline{r}}^{(x, z), \frac{1}{N}}\left(\underline{x}^{\prime}\right)\right) f\left(\underline{k}^{\prime}\right)-g\left(\frac{1}{N}\right) \phi(\underline{\underline{0}}) f(\underline{k})\right\}\right| \\
& \leq\left(\bar{\beta}+\frac{1}{N} \bar{\gamma}_{d}\right) \cdot\|\phi f\| \cdot N\left|g\left(\frac{1}{N}\right)\right| \\
& \quad+\left(\bar{\beta}+\frac{1}{N} \bar{\gamma}_{b}\right) \cdot\|\phi f\| \cdot N\left|g\left(\frac{2}{N}\right)-g\left(\frac{1}{N}\right)\right|+2\left(\bar{\beta}+\frac{1}{N} \bar{\gamma}_{b}\right) \cdot\|\phi f\| \cdot N\left|g\left(\frac{1}{N}\right)\right| \\
& \underset{N \rightarrow \infty}{\longrightarrow} 0
\end{aligned}
$$

where in the last line we made use once more of the assumption that $g^{\prime}(0)=0$.

Assume next that $\chi=\overline{(X, r, \mu)} \in \mathbb{M}_{N}^{K}$ is such that $m(\chi) \geq \frac{2}{N}$. Then (recall (2.7), (3.1) and (4.1))

$$
\begin{aligned}
\Omega_{N} F(\chi) & =\Omega_{N}^{\text {death }} F(\chi)+\Omega_{N}^{\text {birth }} F(\chi) \\
= & N m(\chi) \int_{(X \times K)^{2}} \bar{\mu}^{\otimes 2}\left(\mathrm{~d}\left(\left(x_{1}, \kappa_{1}\right),\left(x_{2}, \kappa_{2}\right)\right)\right)\left\{N \beta\left(\kappa_{2}\right)+\gamma^{\text {death }}\left(m(\chi), r\left(x_{1}, x_{2}\right), \kappa_{1}, \kappa_{2}\right)\right\} \\
& \cdot\left(g\left(m(\chi)-\frac{1}{N}\right) \int_{(X \times K)^{n}}\left(\frac{N m(\chi) \cdot \bar{\mu}-\delta_{\left(x_{2}, \kappa_{2}\right)}}{N m(\chi)-1}\right)^{\otimes n}(\mathrm{~d}(\underline{x}, \underline{\kappa})) \phi(\underline{\underline{r}}(\underline{x})) \cdot f(\underline{\kappa})-g(m(\chi)) \cdot F^{1,(n, \phi, f)}(\chi)\right) \\
+ & N m(\chi) \int_{(X \times K)^{2}} \bar{\mu}^{\otimes 2}\left(\mathrm{~d}\left(\left(x_{1}, \kappa_{1}\right),\left(x_{2}, \kappa_{2}\right)\right)\right)\left\{N \beta\left(\kappa_{2}\right)+\gamma^{\operatorname{birth}}\left(m(\chi), r\left(x_{1}, x_{2}\right), \kappa_{1}, \kappa_{2}\right)\right\} \int_{K} \widehat{\alpha}_{N}\left(\kappa_{2}, \mathrm{~d} \tilde{\kappa}_{2}\right) \\
& \cdot\left(g\left(m(\chi)+\frac{1}{N}\right) \int_{(X \uplus\{z\} \times K)^{n}}\left(\frac{N m(x) \cdot \bar{\mu}+\delta_{\left(z, \tilde{\kappa}_{2}\right)}}{N m(x)+1}\right)^{\otimes n}(\mathrm{~d}(\underline{x}, \underline{\kappa})) \phi\left(\underline{r}^{\left(x_{2}, z\right), \frac{1}{N}}(\underline{x})\right) \cdot f(\underline{\kappa})-g(m(\chi)) \cdot F^{1,(n, \phi, f)}(\chi)\right),
\end{aligned}
$$

with $r^{\left(x_{2}, z\right), \frac{1}{N}}$ as defined in (2.22). We make use of a Taylor expansion. Namely, for all $g \in$ $\mathcal{C}_{b}^{3}\left(\mathbb{R}_{+}\right), m \geq \frac{1}{N}$,

$$
g\left(m \pm \frac{1}{N}\right)=g(m) \pm \frac{1}{N} \cdot g^{\prime}(m)+\frac{1}{2 N^{2}} \cdot g^{\prime \prime}(m)+\mathcal{O}\left(N^{-3}\right) C_{g}(m, N) .
$$

with

$$
C_{g}(m, N):=\max _{\xi \in[m-1 / N, m+1 / N]}\left|g^{\prime \prime \prime}(\xi)\right|
$$


We thereby obtain by Assumptions 1 and 3 ,

$$
\begin{aligned}
& \Omega_{N}^{\text {death }} F^{g,(n, \phi, f)}(\chi) \\
& =g(m(\chi)) \cdot \Omega_{N}^{\text {death }} F^{1,(n, \phi, f)}(\chi) \\
& +m(\chi)\left(-g^{\prime}(m(\chi))+\frac{1}{2 N} g^{\prime \prime}(m(\chi))\right) \int_{(X \times K)^{2}} \bar{\mu}^{\otimes 2}\left(\mathrm{~d}\left(\left(x_{1}, \kappa_{1}\right),\left(x_{2}, \kappa_{2}\right)\right)\right) \\
& \quad \cdot\left\{N \beta\left(\kappa_{2}\right)+\gamma^{\text {death }}\left(m(\chi), r\left(x_{1}, x_{2}\right), \kappa_{1}, \kappa_{2}\right)\right\} \cdot \int_{(X \times K)^{n}}\left(\frac{N m(\chi) \cdot \bar{\mu}-\delta_{\left(x_{2}, \kappa_{2}\right)}}{N m(\chi)-1}\right)^{\otimes n}(\mathrm{~d}(\underline{x}, \underline{\kappa})) \phi(\underline{\underline{r}}(\underline{x})) \cdot f(\underline{\kappa}) \\
& +m(\chi) \cdot\left(\bar{\beta}+\frac{1}{N} \tilde{\gamma}(m(\chi))\right) \cdot C_{g}(m(\chi), N) \cdot \mathcal{O}\left(\frac{1}{N}\right) .
\end{aligned}
$$

Moreover, by Assumptions 1 and 2 (distinguishing between clones and mutants),

$$
\begin{aligned}
& \Omega_{N}^{\text {birth }} F^{g,(n, \phi, f)}(\chi) \\
& =g(m(\chi)) \cdot \Omega_{N}^{\mathrm{birth}} F^{1,(n, \phi, f)}(\chi) \\
& \quad+m(\chi)\left(g^{\prime}(m(\chi))+\frac{1}{2 N} g^{\prime \prime}(m(\chi))\right) \int_{(X \times K)^{2}} \bar{\mu}^{\otimes 2}\left(\mathrm{~d}\left(\left(x_{1}, \kappa_{1}\right),\left(x_{2}, \kappa_{2}\right)\right)\right)\left\{N \beta\left(\kappa_{2}\right)+\gamma^{\operatorname{birth}}\left(m(\chi), r\left(x_{1}, x_{2}\right), \kappa_{1}, \kappa_{2}\right)\right\} \\
& \quad+\left((1-p) \int_{(X \times K)^{n}}\left(\frac{N m(\chi) \cdot \bar{\mu}+\delta_{\left(x_{2}, \kappa_{2}\right)}}{N m(\chi)+1}\right)^{\otimes n}(\mathrm{~d}(\underline{x}, \underline{\kappa})) \phi(\underline{\underline{r}}(\underline{x})) \cdot f(\underline{\kappa})\right. \\
& \left.\quad+p \int_{K} \alpha_{N}\left(\kappa_{2}, \mathrm{~d} \tilde{\kappa}_{2}\right) \int_{(X \uplus\{z\} \times K)^{n}}\left(\frac{N m(\chi) \cdot \bar{\mu}+\delta_{\left(z, \tilde{\kappa}_{2}\right)}}{N m(\chi)+1}\right)^{\otimes n}(\mathrm{~d}(\underline{x}, \underline{\kappa})) \phi\left(\underline{\underline{r}}^{\left(x_{2}, z\right), \frac{1}{N}}(\underline{x})\right) \cdot f(\underline{\kappa})\right) \\
& \quad+m(\chi) \cdot\left(\bar{\beta}+\frac{1}{N} \gamma_{b}\right) \cdot C_{g}(m(\chi), N) \cdot \mathcal{O}\left(\frac{1}{N}\right) .
\end{aligned}
$$

We abbreviate

$$
\begin{aligned}
a & =a_{N}(m(\chi)):=\frac{1}{N} g^{\prime}(m(\chi)) \\
b & =b_{N}(m(\chi)):=\frac{1}{2 N^{2}} \cdot g^{\prime \prime}(m(\chi)) \\
A & =A_{N}\left(m(\chi), \kappa_{2}\right):=N^{2} m(\chi) \cdot \beta\left(\kappa_{2}\right) \\
B & =B_{N}\left(m(\chi), x_{2}, \kappa_{2}\right):=N m(\chi) \cdot \int \bar{\mu}\left(\mathrm{d}\left(x_{1}, \kappa_{1}\right)\right) \gamma^{\operatorname{birth}}\left(m(\chi), r\left(x_{1}, x_{2}\right), \kappa_{1}, \kappa_{2}\right) \\
C & =C_{N}\left(m(\chi), x_{2}, \kappa_{2}\right):=N m(\chi) \cdot \int_{\mu} \bar{\mu}\left(\mathrm{d}\left(x_{1}, \kappa_{1}\right)\right) \gamma^{\operatorname{death}}\left(m(\chi), r\left(x_{1}, x_{2}\right), \kappa_{1}, \kappa_{2}\right) \\
D & =D_{N}\left(m(\chi), x_{2}, \kappa_{2}\right):=(1-p) \int_{(X \times K)^{n}}\left(\frac{N m(\chi) \cdot \bar{\mu}+\delta_{\left(x_{2}, \kappa_{2}\right)}}{N m(\chi)+1}\right)^{\otimes n}(\mathrm{~d}(\underline{x}, \underline{\kappa})) \phi(\underline{\underline{r}}(\underline{x})) \cdot f(\underline{\kappa}) \\
& +p \int_{K} \alpha_{N}\left(\kappa_{2}, \mathrm{~d} \tilde{\kappa}_{2}\right) \int_{(X \uplus\{z\} \times K)^{n}}\left(\frac{N m(\chi) \cdot \bar{\mu}+\delta_{\left(z, \tilde{\kappa}_{2}\right)}}{N m(\chi)+1}\right)^{\otimes n}(\mathrm{~d}(\underline{x}, \underline{\kappa})) \phi\left(\underline{\underline{r}}\left(x_{2}, z\right), \frac{1}{N}(\underline{x})\right) \cdot f(\underline{\kappa}) \\
E & =E_{N}\left(m(\chi), x_{2}, \kappa_{2}\right):=\int_{(X \times K)^{n}}\left(\frac{N m(x) \cdot \bar{\mu}-\delta_{\left(x_{2}, \kappa_{2}\right)}}{N m(\chi)-1}\right)^{\otimes n}(\mathrm{~d}(\underline{x}, \underline{\kappa})) \phi(\underline{\underline{r}}(\underline{x})) \cdot f(\underline{\kappa}) .
\end{aligned}
$$


Rewrite

$$
\begin{aligned}
& \Omega_{N}^{\text {death }} F^{g,(n, \phi, f)}(\chi) \\
& =g(m(\chi)) \cdot \Omega_{N}^{\text {death }} F^{1,(n, \phi, f)}(\chi)+(-a+b) \cdot \int_{X \times K} \bar{\mu}\left(\mathrm{d}\left(x_{2}, \kappa_{2}\right)\right)\{A+C\} \cdot E \\
& +m(\chi) \cdot\left(\bar{\beta}+\frac{1}{N} \tilde{\gamma}(m(\chi))\right) C_{g}(m(\chi), N) \mathcal{O}\left(\frac{1}{N}\right),
\end{aligned}
$$

and

$$
\begin{aligned}
& \Omega_{N}^{\text {birth }} F^{g,(n, \phi, f)}(\chi) \\
& =g(m(\chi)) \cdot \Omega_{N}^{\text {birth }} F^{1,(n, \phi, f)}(\chi)+(a+b) \cdot \int_{X \times K} \bar{\mu}\left(\mathrm{d}\left(x_{2}, \kappa_{2}\right)\right)\{A+B\} \cdot D \\
& +m(\chi) \cdot\left(\bar{\beta}+\frac{1}{N} \gamma_{b}\right) C_{g}(m(\chi), N) \mathcal{O}\left(\frac{1}{N}\right) .
\end{aligned}
$$

As

$$
\begin{aligned}
& (a+b)\{A+B\} D-(a-b)\{A+C\} E \\
& =a A(D-E)+a(B-C) E+b A(D+E)+a B(D-E)+b\{B \cdot D+C \cdot E\},
\end{aligned}
$$

this yields

$$
\begin{aligned}
& \Omega_{N} F^{g,(n, \phi, f)}(\chi) \\
& =g(m(\chi)) \cdot \Omega_{N} F^{1,(n, \phi, f)}(\chi) \\
& +a \int \mathrm{d} \bar{\mu} A(D-E)+a \int \mathrm{d} \bar{\mu}(B-C) E+b \cdot \int \mathrm{d} \bar{\mu} A(D+E) \\
& +a \int \mathrm{d} \bar{\mu} B(D-E)+b \int \mathrm{d} \bar{\mu}\{B \cdot D+C \cdot E\}+m(\chi) \cdot\left(1 \vee \frac{\tilde{\gamma}(m(x))}{N}\right) C_{g}(m(\chi), N) \cdot \mathcal{O}\left(\frac{1}{N}\right) \\
& =: g(m(\chi)) \cdot T_{1}+T_{2}+E_{3} .
\end{aligned}
$$

In what follows we analyse each of the three terms separately.

Step 0 (Preparatory calculations) For all $n \geq 2$ fixed, $N \cdot m \geq 2$,

$$
\begin{aligned}
& \left(\frac{N m \bar{\mu} \pm \delta_{(z, \kappa)}}{N m \pm 1}\right)^{\otimes n} \\
& =\left(\bar{\mu} \pm \frac{\left(\delta_{(z, \kappa)}-\bar{\mu}\right)}{N m \pm 1}\right)^{\otimes n} \\
& =\bar{\mu}^{\otimes n} \pm \sum_{l=1}^{n} \bar{\mu}^{\otimes(l-1)} \otimes\left(\frac{\delta_{(z, \kappa)}-\bar{\mu}}{N m \pm 1}\right) \otimes \bar{\mu}^{\otimes(n-l)} \\
& +\sum_{1 \leq l_{1}<l_{2} \leq n} \bar{\mu}^{\otimes\left(l_{1}-1\right)} \otimes\left(\frac{\delta_{(z, \kappa)}-\bar{\mu}}{N m \pm 1}\right) \otimes \bar{\mu}^{\otimes\left(l_{2}-l_{1}-1\right)} \otimes\left(\frac{\delta_{(z, \kappa)}-\bar{\mu}}{N m \pm 1}\right) \otimes \bar{\mu}^{\otimes\left(n-l_{2}\right)}+\mathcal{O}\left((N m)^{-3}\right),
\end{aligned}
$$


which we rewrite to

$$
\begin{aligned}
& \left(\frac{N m \bar{\mu} \pm \delta_{(z, \kappa)}}{N m \pm 1}\right)^{\otimes n}-\bar{\mu}^{\otimes n} \\
& =\frac{n}{N m \pm 1}\left(\mp 1+\frac{(n-1)}{2(N m \pm 1)}\right) \bar{\mu}^{\otimes n} \\
& +\frac{1}{N m \pm 1}\left( \pm 1-\frac{n-1}{(N m \pm 1)}\right) \sum_{l=1}^{n} \bar{\mu}^{\otimes(l-1)} \otimes \delta_{(z, \kappa)} \otimes \bar{\mu}^{\otimes(n-l)} \\
& +\frac{1}{(N m \pm 1)^{2}} \sum_{1 \leq l_{1}<l_{2} \leq n} \bar{\mu}^{\otimes\left(l_{1}-1\right)} \otimes \delta_{(z, \kappa)} \otimes \bar{\mu}^{\otimes\left(l_{2}-l_{1}-1\right)} \otimes \delta_{(z, \kappa)} \otimes \bar{\mu}^{\otimes\left(n-l_{2}\right)}+\mathcal{O}\left((N m)^{-3}\right) \\
& =-\frac{n(n-1)}{2(N m \pm 1)^{2}} \bar{\mu}^{\otimes n}+\frac{1}{N m \pm 1}\left( \pm 1-\frac{n-1}{(N m \pm 1)}\right) \sum_{l=1}^{n} \bar{\mu}^{\otimes(l-1)} \otimes\left(\delta_{(z, \kappa)}-\bar{\mu}\right) \otimes \bar{\mu}^{\otimes(n-l)} \\
& +\frac{1}{(N m \pm 1)^{2}} \sum_{1 \leq l_{1}<l_{2} \leq n} \bar{\mu}^{\otimes\left(l_{1}-1\right)} \otimes \delta_{(z, \kappa)} \otimes \bar{\mu}^{\otimes\left(l_{2}-l_{1}-1\right)} \otimes \delta_{(z, \kappa)} \otimes \bar{\mu}^{\otimes\left(n-l_{2}\right)}+\mathcal{O}\left((N m)^{-3}\right) .
\end{aligned}
$$

This implies that

$$
\begin{aligned}
\left(\frac{N m \bar{\mu}+\delta_{(z, \kappa)}}{N m+1}\right)^{\otimes n}+\left(\frac{N m \bar{\mu}-\delta_{(z, \kappa)}}{N m-1}\right)^{\otimes n}-2 \bar{\mu}^{\otimes n} \\
=-\frac{n(n-1)}{2}\left(\frac{1}{(N m+1)^{2}}+\frac{1}{(N m-1)^{2}}\right) \bar{\mu}^{\otimes n} \\
\quad+\left(\frac{1}{N m+1}\left(1-\frac{n-1}{(N m+1)}\right)-\frac{1}{N m-1}\left(1+\frac{n-1}{(N m-1)}\right)\right) \sum_{l=1}^{n} \bar{\mu}^{\otimes(l-1)} \otimes\left(\delta_{(z, \kappa)}-\bar{\mu}\right) \otimes \bar{\mu}^{\otimes(n-l)} \\
\quad+\left(\frac{1}{(N m+1)^{2}}+\frac{1}{(N m-1)^{2}}\right) \sum_{1 \leq l_{1}<l_{2} \leq n} \bar{\mu}^{\otimes\left(l_{1}-1\right)} \otimes \delta_{(z, \kappa)} \otimes \bar{\mu}^{\otimes\left(l_{2}-l_{1}-1\right)} \otimes \delta_{(z, \kappa)} \otimes \bar{\mu}^{\otimes\left(n-l_{2}\right)}+\mathcal{O}\left((N m)^{-3}\right) \\
=-\frac{n(n-1)\left((N m)^{2}+1\right)}{\left((N m)^{2}-1\right)^{2}} \bar{\mu}^{\otimes n} \\
\quad+\frac{1}{\left((N m)^{2}-1\right)^{2}}\left(-2\left((N m)^{2}-1\right)-2(n-1)\left((N m)^{2}+1\right)\right) \sum_{l=1}^{n} \bar{\mu}^{\otimes(l-1)} \otimes\left(\delta_{(z, \kappa)}-\bar{\mu}\right) \otimes \bar{\mu}^{\otimes(n-l)} \\
\quad+\frac{(N m-1)^{2}+(N m+1)^{2}}{\left((N m)^{2}-1\right)^{2}} \sum_{1 \leq l_{1}<l_{2} \leq n} \bar{\mu}^{\otimes\left(l_{1}-1\right)} \otimes \delta_{(z, \kappa)} \otimes \bar{\mu}^{\otimes\left(l_{2}-l_{1}-1\right)} \otimes \delta_{(z, \kappa)} \otimes \bar{\mu}^{\otimes\left(n-l_{2}\right)}+\mathcal{O}\left((N m)^{-3}\right) .
\end{aligned}
$$

Thus

$$
\begin{aligned}
& \left(\frac{N m \bar{\mu}+\delta_{(z, \kappa)}}{N m+1}\right)^{\otimes n}+\left(\frac{N m \bar{\mu}-\delta_{(z, \kappa)}}{N m-1}\right)^{\otimes n}-2 \bar{\mu}^{\otimes n} \\
& =-\frac{2 n}{(N m)^{2}} \sum_{l=1}^{n} \bar{\mu}^{\otimes(l-1)} \otimes\left(\delta_{(z, \kappa)}-\bar{\mu}\right) \otimes \bar{\mu}^{\otimes(n-l)} \\
& \quad+\frac{2}{(N m)^{2}} \sum_{1 \leq l_{1}<l_{2} \leq n}\left(\bar{\mu}^{\otimes\left(l_{1}-1\right)} \otimes \delta_{(z, \kappa)} \otimes \bar{\mu}^{\otimes\left(l_{2}-l_{1}-1\right)} \otimes \delta_{(z, \kappa)} \otimes \bar{\mu}^{\otimes\left(n-l_{2}\right)}-\bar{\mu}^{\otimes n}\right)+\mathcal{O}\left((N m)^{-3}\right) .
\end{aligned}
$$


Formula (4.19) also yields

$$
\begin{aligned}
& \left(\frac{N m \bar{\mu}+\delta_{(z, \kappa)}}{N m+1}\right)^{\otimes n}-\left(\frac{N m \bar{\mu}-\delta_{(\tilde{z}, \tilde{\kappa})}}{N m-1}\right)^{\otimes n} \\
& =\sum_{l=1}^{n} \bar{\mu}^{\otimes(l-1)} \otimes\left(\frac{\delta_{(z, \kappa)}-\bar{\mu}}{N m+1}\right) \otimes \bar{\mu}^{\otimes(n-l)}+\sum_{l=1}^{n} \bar{\mu}^{\otimes(l-1)} \otimes\left(\frac{\delta_{(\tilde{z}, \tilde{\kappa})}-\bar{\mu}}{N m-1}\right) \otimes \bar{\mu}^{\otimes(n-l)} \\
& +\sum_{1 \leq l_{1}<l_{2} \leq n} \bar{\mu}^{\otimes\left(l_{1}-1\right)} \otimes\left(\frac{\delta_{(z, \kappa)}-\bar{\mu}}{N m+1}\right) \otimes \bar{\mu}^{\otimes\left(l_{2}-l_{1}-1\right)} \otimes\left(\frac{\delta_{(z, \kappa)}-\bar{\mu}}{N m+1}\right) \otimes \bar{\mu}^{\otimes\left(n-l_{2}\right)} \\
& \quad-\sum_{1 \leq l_{1}<l_{2} \leq n} \bar{\mu}^{\otimes\left(l_{1}-1\right)} \otimes\left(\frac{\delta_{(\tilde{z}, \tilde{\kappa})}-\bar{\mu}}{N m-1}\right) \otimes \bar{\mu}^{\otimes\left(l_{2}-l_{1}-1\right)} \otimes\left(\frac{\delta_{(\tilde{z}, \tilde{\kappa})}-\bar{\mu}}{N m-1}\right) \otimes \bar{\mu}^{\otimes\left(n-l_{2}\right)}+\mathcal{O}\left((N m)^{-3}\right), \\
& =\frac{1}{N m} \sum_{l=1}^{n} \bar{\mu}^{\otimes(l-1)} \otimes\left(\delta_{(z, \kappa)}+\delta_{(\tilde{z}, \tilde{\kappa})}-2 \bar{\mu}\right) \otimes \bar{\mu}^{\otimes(n-l)}+\mathcal{O}\left((N m)^{-2}\right) .
\end{aligned}
$$

Step 1 (The term $T_{1}$ ) Recall from (4.18) the term $T_{1}=\Omega_{N} F^{1,(n, \phi, f)}(\chi)$. This term describes the changes we see once we force the total mass to be constant. Recall from (4.9) that $\Omega_{N}=$ $\Omega_{N}^{\text {death }}+\Omega_{N}^{\text {birth }}$. We start with the death part which we split in natural death which happens on a faster time scale and death due to competition which occurs more rarely:

$$
\begin{aligned}
& \Omega_{N}^{\text {death }} F^{1,(n, \phi, f)}(\chi) \\
& =: \Omega_{N}^{\text {natural death }} F^{1,(n, \phi, f)}(\chi)+\Omega_{N}^{\text {competition }} F^{1,(n, \phi, f)}(\chi) \\
& =N^{2} m(\chi) \int_{(X \times K)^{2}} \bar{\mu}^{\otimes 2}\left(\mathrm{~d}\left(\left(x_{1}, \kappa_{1}\right),\left(x_{2}, \kappa_{2}\right)\right)\right) \beta\left(\kappa_{2}\right) \\
& \quad \cdot \int_{(X \times K)^{n}}\left(\left(\frac{N m(\chi) \cdot \bar{\mu}-\delta_{\left(x_{2}, \kappa_{2}\right)}}{N m(\chi)-1}\right)^{\otimes n}-\bar{\mu}^{\otimes n}\right)(\mathrm{d}(\underline{x}, \underline{\kappa})) \phi(\underline{\underline{r}}(\underline{x})) \cdot f(\underline{\kappa}) \\
& +N m(\chi) \int_{(X \times K)^{2}} \bar{\mu}^{\otimes 2}\left(\mathrm{~d}\left(\left(x_{1}, \kappa_{1}\right),\left(x_{2}, \kappa_{2}\right)\right)\right) \gamma^{\operatorname{death}}\left(m(\chi), r\left(x_{1}, x_{2}\right), \kappa_{1}, \kappa_{2}\right) \\
& \quad \cdot \int_{(X \times K)^{n}}\left(\left(\frac{N m(\chi) \cdot \bar{\mu}-\delta_{\left(x_{2}, \kappa_{2}\right)}}{N m(\chi)-1}\right)^{\otimes n}-\bar{\mu}^{\otimes n}\right)(\mathrm{d}(\underline{x}, \underline{\kappa})) \phi(\underline{r}(\underline{x})) \cdot f(\underline{\kappa}) .
\end{aligned}
$$

For the birth-part, we have to take into consideration possible mutation events. Thus $(4.25)$

$$
\begin{aligned}
& \Omega_{N}^{\text {birth }} F^{1,(n, \phi, f)}(\chi) \\
& =:(1-p) \cdot \Omega_{N}^{\text {birth;no mutation }} F^{1,(n, \phi, f)}(\chi)+p \cdot \Omega_{N}^{\text {birth;mutation }} F^{1,(n, \phi, f)}(\chi) \\
& =N m(\chi) \int_{(X \times K)^{2}} \bar{\mu}^{\otimes 2}\left(\mathrm{~d}\left(\left(x_{1}, \kappa_{1}\right),\left(x_{2}, \kappa_{2}\right)\right)\right)\left\{N \beta\left(\kappa_{2}\right)+\gamma^{\text {birth }}\left(m(\chi), r\left(x_{1}, x_{2}\right), \kappa_{1}, \kappa_{2}\right)\right\} \\
& \cdot\left\{(1-p) \cdot \int_{(X \times K)^{n}}\left(\left(\frac{N m(\chi) \cdot \bar{\mu}+\delta_{\left(x_{2}, \kappa_{2}\right)}}{N m(\chi)+1}\right)^{\otimes n}-\bar{\mu}^{\otimes n}\right)(\mathrm{d}(\underline{x}, \underline{\kappa})) \phi(\underline{\underline{r}}(\underline{x})) \cdot f(\underline{\kappa})\right. \\
& \left.\left.\quad+p \int_{K} \alpha_{N}\left(\kappa_{2}, \mathrm{~d} \tilde{\kappa}_{2}\right) \int_{(X \uplus\{z\} \times K)^{n}}\left(\left(\frac{N m(\chi) \cdot \bar{\mu}+\delta_{\left(z, \tilde{\kappa}_{2}\right)}}{N m(\chi)+1}\right)^{\otimes n}-\bar{\mu}^{\otimes n}\right)(\mathrm{d}(\underline{x}, \underline{\kappa})) \phi\left(\underline{\underline{r}}^{\left(x_{2}, z\right), \frac{1}{N}}(\underline{x})\right) \cdot f(\underline{\kappa})\right)\right\} .
\end{aligned}
$$


We also distinguish for each of the birth events (with and without mutation) between natural birth events which happen at a fast time scale and enhancement which occurs more rarely. That is, we use the notation (with the terms obtained similar as in (4.24))

$$
\begin{aligned}
\Omega_{N}^{\text {birth }}= & :(1-p) \cdot \Omega_{N}^{\text {natural birth; no mutation }}+(1-p) \cdot \Omega_{N}^{\text {enhancement; no mutation }} \\
& +p \cdot \Omega_{N}^{\text {natural birth; mutation }}+p \cdot \Omega_{N}^{\text {enhancement; mutation }} .
\end{aligned}
$$

We therefore base our study of $T_{1}$ on the following decomposition:

$$
\begin{aligned}
T_{1}= & \left(\Omega_{N}^{\text {enhancement; no mutation }}+\Omega_{N}^{\text {competition }}\right) F^{1,(n, \phi, f)}(\chi) \\
& +\left(\Omega_{N}^{\text {natural birth; no mutation }}+\Omega_{N}^{\text {natural death }}\right) F^{1,(n, \phi, f)}(\chi) \\
& +p \cdot\left(\Omega_{N}^{\text {natural birth; mutation }}-\Omega_{N}^{\text {natural birth; no mutation }}\right) F^{1,(n, \phi, f)}(\chi) \\
& +p \cdot\left(\Omega_{N}^{\text {enhancement; mutation }}-\Omega_{N}^{\text {enhancement; no mutation }}\right) F^{1,(n, \phi, f)}(\chi) \\
= & : T_{1.1}+T_{1.2}+T_{1.3}+E_{1.4} .
\end{aligned}
$$

Once more we are handling the different terms separately. Recall the sequence $\left(a_{N}\right)$ from (4.5).

Step 1.1 (Reweighing the sampling measure with respect to $\Gamma$ ) Recall $\Gamma$ from (2.35), and $\Omega_{\Gamma \text {-reweigh }}^{\Gamma}$ from (2.44). By (4.24) and (4.25),

$$
\begin{aligned}
& T_{1.1}=N m(\chi) \int_{(X \times K)^{2}} \bar{\mu}^{\otimes 2}\left(\mathrm{~d}\left(\left(x_{1}, \kappa_{1}\right),\left(x_{2}, \kappa_{2}\right)\right)\right) \gamma^{\text {death }}\left(m(\chi), r\left(x_{1}, x_{2}\right), \kappa_{1}, \kappa_{2}\right) \\
& \cdot \int_{(X \times K)^{n}}\left(\left(\frac{N m(\chi) \cdot \bar{\mu}-\delta_{\left(x_{2}, \kappa_{2}\right)}}{N m(\chi)-1}\right)^{\otimes n}-\bar{\mu}^{\otimes n}\right)(\mathrm{d}(\underline{x}, \underline{\kappa})) \phi(\underline{\underline{r}}(\underline{x})) \cdot f(\underline{\kappa}) \\
& +N m(\chi) \int_{(X \times K)^{2}} \bar{\mu}^{\otimes 2}\left(\mathrm{~d}\left(\left(x_{1}, \kappa_{1}\right),\left(x_{2}, \kappa_{2}\right)\right)\right) \gamma^{\operatorname{birth}}\left(m(\chi), r\left(x_{1}, x_{2}\right), \kappa_{1}, \kappa_{2}\right) \\
& \cdot \int_{(X \times K)^{n}}\left(\left(\frac{N m(\chi) \cdot \bar{\mu}+\delta_{\left(x_{2}, \kappa_{2}\right)}}{N m(\chi)+1}\right)^{\otimes n}-\bar{\mu}^{\otimes n}\right)(\mathrm{d}(\underline{x}, \underline{\kappa})) \phi(\underline{\underline{r}}(\underline{x})) \cdot f(\underline{\kappa}) \\
& =N m(\chi) \int_{(X \times K)^{2}} \bar{\mu}^{\otimes 2}\left(\mathrm{~d}\left(\left(x_{1}, \kappa_{1}\right),\left(x_{2}, \kappa_{2}\right)\right)\right) \gamma^{\text {death }}\left(m(\chi), r\left(x_{1}, x_{2}\right), \kappa_{1}, \kappa_{2}\right) \\
& \cdot \int_{(X \times K)^{n}}\left(\left(\frac{N m(\chi) \cdot \bar{\mu}-\delta_{\left(x_{2}, \kappa_{2}\right)}}{N m(\chi)-1}\right)^{\otimes n}+\left(\frac{N m(\chi) \cdot \bar{\mu}+\delta_{\left(x_{2}, \kappa_{2}\right)}}{N m(\chi)+1}\right)^{\otimes n}-2 \bar{\mu}^{\otimes n}\right)(\mathrm{d}(\underline{x}, \underline{\kappa})) \phi(\underline{\underline{r}}(\underline{x})) \cdot f(\underline{\kappa}) \\
& +N m(\chi) \int_{(X \times K)^{2}} \bar{\mu}^{\otimes 2}\left(\mathrm{~d}\left(\left(x_{1}, \kappa_{1}\right),\left(x_{2}, \kappa_{2}\right)\right)\right) \Gamma\left(m(\chi), r\left(x_{1}, x_{2}\right), \kappa_{1}, \kappa_{2}\right) \\
& \cdot \int_{(X \times K)^{n}}\left(\left(\frac{N m(\chi) \cdot \bar{\mu}+\delta_{\left(x_{2}, \kappa_{2}\right)}}{N m(\chi)+1}\right)^{\otimes n}-\bar{\mu}^{\otimes n}\right)(\mathrm{d}(\underline{x}, \underline{\kappa})) \phi(\underline{\underline{r}}(\underline{x})) \cdot f(\underline{\kappa}) .
\end{aligned}
$$


Thus by (4.22) and (4.20) together with Assumption 3 ,

$$
\begin{aligned}
T_{1.1}= & (1 \vee \tilde{\gamma}(m(\chi))) \cdot \mathcal{O}\left((N m(\chi))^{-1}\right) \\
+ & \int_{(X \times K)^{2}} \bar{\mu}^{\otimes 2}\left(\mathrm{~d}\left(\left(x_{1}, \kappa_{1}\right),\left(x_{2}, \kappa_{2}\right)\right)\right) \Gamma\left(m(\chi), r\left(x_{1}, x_{2}\right), \kappa_{1}, \kappa_{2}\right) \\
& \quad \cdot \sum_{l=1}^{n} \int_{(X \times K)^{n}} \bar{\mu}^{\otimes(l-1)} \otimes\left(\delta_{\left(x_{2}, \kappa_{2}\right)}-\bar{\mu}\right) \otimes \bar{\mu}^{\otimes(n-l)}(\mathrm{d}(\underline{x}, \underline{\kappa})) \phi(\underline{\underline{r}}(\underline{x})) \cdot f(\underline{\kappa}) \\
= & \Omega_{\Gamma \text {-reweigh }}^{\Gamma} F^{1,(n, \phi, f)}(\chi)+(1 \vee \tilde{\gamma}(m(\chi))) \cdot \mathcal{O}\left(\left(N m(\chi)^{-1}\right) .\right.
\end{aligned}
$$

Hence, for some $C \in(0, \infty)$,

$$
\begin{aligned}
& \sup _{\chi \in \mathbb{M}_{N}^{K} ; \frac{2}{N} \leq m(\chi) \leq \frac{a_{N}}{N}}\left|g(m(\chi)) \cdot T_{1.1}-\Omega_{\Gamma \text {-reweigh }}^{\Gamma} F^{g,(n, \phi, f)}(\chi)\right| \\
& \leq C \cdot \sup _{m \in\left[\frac{2}{N}, \frac{a_{N}}{N}\right]} \frac{|g(m)|}{m} \frac{(1 \vee \tilde{\gamma}(m))}{N} \underset{N \rightarrow \infty}{\longrightarrow} 0,
\end{aligned}
$$

as $g$ is bounded differentiable at $m=0$ and $\tilde{g}$ is continuous. Moreover,

$$
\begin{aligned}
& \sup _{\chi \in \mathbb{M}_{N}^{K} ; m(\chi) \geq \frac{a_{N}}{N}}\left|g(m(\chi)) \cdot T_{1.1}-\Omega_{\Gamma-\text { reweigh }}^{\Gamma} F^{g,(n, \phi, f)}(\chi)\right| \\
& \leq C \cdot a_{N}^{-1} \cdot \sup _{m \in\left[\frac{a_{N}}{N}, \infty\right)}|g(m)|(1 \vee \tilde{\gamma}(m)) \underset{N \rightarrow \infty}{\longrightarrow} 0,
\end{aligned}
$$

as $\lim \sup _{m \rightarrow \infty}(1 \vee \tilde{\gamma}(m))|g(m)|<\infty($ compare (4.3) $)$ and $a_{N} \rightarrow \infty$ for $N \rightarrow \infty$.

Step 1.2 (Effect of natural branching on the genealogy) Recall $\widehat{\beta}^{x}$ from (2.38), and the replacement map $\Theta_{l_{1}, l_{2}}$ from (2.47) and (2.48). Recall further $\Omega_{\beta \text {-natural branching }}^{\beta}, \Omega_{\beta \text {-reweigh }}^{\beta}$ and $\Omega_{\text {resample }}^{\beta}$ from (2.46). By (4.24) and (4.25),

$$
\begin{aligned}
& T_{1.2} \\
& =N^{2} m(\chi) \int_{X \times K} \bar{\mu}\left(\mathrm{d}\left(x^{\prime}, \kappa^{\prime}\right)\right) \beta\left(\kappa^{\prime}\right) \\
& \quad \cdot \int_{(X \times K)^{n}}\left(\left(\frac{N m(x) \cdot \bar{\mu}-\delta_{\left(x^{\prime}, \kappa^{\prime}\right)}}{N m(x)-1}\right)^{\otimes n}+\left(\frac{N m(x) \cdot \bar{\mu}+\delta_{\left(x^{\prime}, \kappa^{\prime}\right)}}{N m(x)+1}\right)^{\otimes n}-2 \bar{\mu}^{\otimes n}\right)(\mathrm{d}(\underline{x}, \underline{\kappa})) \phi(\underline{\underline{r}}(\underline{x})) \cdot f(\underline{\kappa}) .
\end{aligned}
$$


Using once more (4.22), we find that (4.33)

$$
\begin{aligned}
T_{1.2}= & -\frac{2 n}{m(x)} \int \sum_{l=1}^{n} \bar{\mu}^{\otimes n}(\mathrm{~d}(\underline{x}, \underline{\kappa}))\left(\beta\left(\kappa_{l}\right)-\widehat{\beta}^{\chi}\right) \cdot \phi(\underline{\underline{r}}(\underline{x})) \cdot f(\underline{\kappa}) \\
& \left.+\frac{1}{m(\chi)} \int \bar{\mu}^{\otimes n}(\mathrm{~d}(\underline{x}, \underline{\kappa})) \sum_{1 \leq l_{1} \neq l_{2} \leq n}\left\{\beta\left(\kappa_{l_{1}}\right) \Theta_{l_{1}, l_{2}}(\phi \cdot f)-\widehat{\beta}^{\chi} \phi \cdot f\right\}(\underline{\underline{r}}, \underline{\kappa})\right\}+N^{2} m(\chi) \cdot \mathcal{O}\left((N m(\chi))^{-3}\right) \\
= & -\frac{1}{m(\chi)} \int \sum_{l=1}^{n} \bar{\mu}^{\otimes n}(\mathrm{~d}(\underline{x}, \underline{\kappa}))\left(\beta\left(\kappa_{l}\right)-\widehat{\beta}^{\chi}\right) \cdot \phi(\underline{\underline{r}}(\underline{x})) \cdot f(\underline{\kappa})+\mathcal{O}\left(N^{-1}(m(\chi))^{-2}\right) \\
& +\frac{1}{m(\chi)} \int \bar{\mu}^{\otimes n}(\mathrm{~d}(\underline{x}, \underline{\kappa})) \sum_{1 \leq l_{1}, l_{2} \leq n}\left\{\beta\left(\kappa_{l_{1}}\right) \cdot\left(\Theta_{l_{1}, l_{2}}(\phi \cdot f)-\phi \cdot f\right)+\left(\widehat{\beta}^{\chi}-\beta\left(\kappa_{l_{2}}\right)\right) \phi \cdot f\right\}(m(\chi), \underline{\underline{r}}, \underline{\kappa}) \\
= & \left(\Omega_{\beta \text {-reweigh }}^{\beta}+\Omega_{\text {resample }}^{\beta}\right) F^{1,(n, \phi, f)}(\chi)+\mathcal{O}\left(N^{-1}(m(\chi))^{-2}\right) \\
= & \Omega_{\beta \text {-natural branching }}^{\beta} F^{1,(n, \phi, f)}(\chi)+\mathcal{O}\left(N^{-1}(m(\chi))^{-2}\right) .
\end{aligned}
$$

Thus for some $C \in(0, \infty)$,

$$
\begin{aligned}
& \sup _{\chi \in \mathbb{M}_{N}^{K} ; \frac{2}{N} \leq m(\chi) \leq \frac{a_{N}}{N}}\left|g(m(\chi)) \cdot T_{1.2}-\Omega_{\text {natural branching }}^{\beta} F^{g,(n, \phi, f)}(\chi)\right| \\
& \leq C \cdot \sup _{m \in\left[\frac{2}{N}, \frac{a_{N}}{N}\right]} \frac{|g(m)|}{m} \frac{1}{N \cdot m} \underset{N \rightarrow \infty}{\longrightarrow} 0,
\end{aligned}
$$

as $g$ is bounded differentiable at $m=0$ and $g^{\prime}(0)=0$. Moreover,

$$
\begin{aligned}
& \sup _{\chi \in \mathbb{M}_{N}^{K} ; m(\chi) \geq \frac{a_{N}}{N}}\left|g(m(\chi)) \cdot T_{1.2}-\Omega_{\text {natural branching }}^{\beta} F^{g,(n, \phi, f)}(\chi)\right| \\
& \leq C \cdot a_{N}^{-1} \cdot \sup _{m \in\left[\frac{a_{N}}{N}, \infty\right)} \frac{|g(m)|}{m} \underset{N \rightarrow \infty}{\longrightarrow} 0,
\end{aligned}
$$

as $\sup _{m>0} \frac{g(m)}{m}<\infty$ and $a_{N} \underset{N \rightarrow \infty}{\longrightarrow} \infty$. 
Step 1.3 (Trait mutation and substitution distance growth) Recall $r^{\left(x_{2}, z\right), l}$ from (2.22) as well as $\Omega_{\text {trait mutation }}^{p, \beta, A}$ and $\Omega_{\text {growth }}^{p, \beta}$ from (2.42) and (2.43). Then by (4.25),

$(4.36)$

$T_{1.3}$

$$
\begin{aligned}
& =p \cdot N^{2} m(\chi) \cdot \int_{X \times K} \bar{\mu}\left(\mathrm{d}\left(x_{2}, \kappa_{2}\right)\right) \beta\left(\kappa_{2}\right) \\
& \cdot\left\{\int_{K} \alpha_{N}\left(\kappa_{2}, \mathrm{~d} \tilde{\kappa}_{2}\right) \int_{(X \uplus\{z\} \times K)^{n}}\left(\left(\frac{N m(\chi) \cdot \bar{\mu}+\delta_{\left(z, \tilde{\kappa}_{2}\right)}}{N m(x)+1}\right)^{\otimes n}-\bar{\mu}^{\otimes n}\right)(\mathrm{d}(\underline{x}, \underline{\kappa})) \phi \underline{\underline{r}}^{\left(x_{2}, z\right), \frac{1}{N}}(\underline{x})\right) \cdot f(\underline{\kappa}) \\
& \left.\left.-\int_{(X \uplus\{z\} \times K)^{n}}\left(\left(\frac{N m(x) \cdot \bar{\mu}+\delta_{\left(x_{2}, \kappa_{2}\right)}}{N m(\chi)+1}\right)^{\otimes n}-\bar{\mu}^{\otimes n}\right)(\mathrm{d}(\underline{x}, \underline{\kappa})) \phi \underline{\underline{r}}^{\left(x_{2}, z\right), \frac{1}{N}}(\underline{x})\right) \cdot f(\underline{\kappa})\right\} \\
& =p \cdot \int_{X \times K} \bar{\mu}\left(\mathrm{d}\left(x_{2}, \kappa_{2}\right)\right) \beta\left(\kappa_{2}\right) \int_{K} N\left(\alpha_{N}\left(\kappa_{2}, \mathrm{~d} \tilde{\kappa}_{2}\right)-\delta\left(\kappa_{2}, \mathrm{~d} \tilde{\kappa}_{2}\right)\right) \\
& \cdot \int_{(X \uplus\{z\} \times K)^{n}} N m(\chi)\left(\left(\frac{N m(\chi) \cdot \bar{\mu}+\delta_{\left(x_{2}, \tilde{\kappa}_{2}\right)}}{N m(\chi)+1}\right)^{\otimes n}-\bar{\mu}^{\otimes n}\right)(\mathrm{d}(\underline{x}, \underline{\kappa})) \phi\left(\underline{\underline{r}}^{\left(x_{2}, z\right), \frac{1}{N}}(\underline{x})\right) \cdot f(\underline{\kappa}) \\
& +p \cdot \int_{X \times K} \bar{\mu}\left(\mathrm{d}\left(x_{2}, \kappa_{2}\right)\right) \beta\left(\kappa_{2}\right) \int_{K} \alpha_{N}\left(\kappa_{2}, \mathrm{~d} \tilde{\kappa}_{2}\right) \\
& \cdot \int_{(X \uplus\{z\} \times K)^{n}} N m(\chi) \cdot\left(\left(\frac{N m(\chi) \cdot \bar{\mu}+\delta_{\left(z, \tilde{\kappa}_{2}\right)}}{N m(\chi)+1}\right)^{\otimes n}-\left(\frac{N m(\chi) \cdot \bar{\mu}+\delta_{\left(x_{2}, \tilde{\kappa}_{2}\right)}}{N m(\chi)+1}\right)^{\otimes n}\right)(\mathrm{d}(\underline{x}, \underline{\kappa})) N \phi\left(\underline{\underline{r}}^{\left(x_{2}, z\right), \frac{1}{N}}(\underline{x})\right) \cdot f(\underline{\kappa}) .
\end{aligned}
$$

Applying (4.20) we find that

$T_{1.3}$

$$
\begin{aligned}
& =p \int_{X \times K} \bar{\mu}\left(\mathrm{d}\left(x_{2}, \kappa_{2}\right)\right) \beta\left(\kappa_{2}\right) \int_{K} N\left(\alpha_{N}\left(\kappa_{2}, \mathrm{~d} \tilde{\kappa}_{2}\right)-\delta\left(\kappa_{2}, \mathrm{~d} \tilde{\kappa}_{2}\right)\right) \\
& \cdot\left\{\int_{(X \uplus\{z\} \times K)^{n}} \sum_{l=1}^{n} \bar{\mu}^{\otimes(l-1)} \otimes\left(\delta_{\left(x_{2}, \tilde{\kappa}_{2}\right)}-\bar{\mu}\right) \otimes \bar{\mu}^{\otimes(n-l)}(\mathrm{d}(\underline{x}, \underline{\kappa})) \phi\left(\underline{\underline{r}}^{\left(x_{2}, z\right), \frac{1}{N}}(\underline{x})\right) \cdot f(\underline{\kappa})+\mathcal{O}\left((N m(\chi))^{-1}\right)\right\} \\
& +p \cdot \int_{X \times K} \bar{\mu}\left(\mathrm{d}\left(x_{2}, \kappa_{2}\right)\right) \beta\left(\kappa_{2}\right) \int_{K} \alpha_{N}\left(\kappa_{2}, \mathrm{~d} \tilde{\kappa}_{2}\right) \\
& \cdot\left\{\int _ { ( X \uplus \{ z \} \times K ) ^ { n } } \sum _ { l = 1 } ^ { n } \overline { \mu } ^ { \otimes ( l - 1 ) } \otimes \left(\delta_{\left(z, \tilde{\kappa}_{2}\right)}-\delta_{\left(x_{2}, \tilde{\kappa}_{2}\right)} \otimes \bar{\mu}^{\otimes(n-l)}(\mathrm{d}(\underline{x}, \underline{\kappa})) \frac{\phi\left(\underline{\underline{r}}^{\left(x_{2}, z\right), \frac{1}{N}}(\underline{x})\right)}{\frac{1}{N}} f(\underline{\kappa})\right.\right. \\
& \left.+\mathcal{O}\left((N m(\chi))^{-1}\right)\right\} \text {. }
\end{aligned}
$$

Recall the mutation operators $A^{(l)} ; l \in\{1, \ldots, n\}$ from (2.42) which act on a function as the mutation operator $A$ on the function of the $l^{\text {th }}$-trait-coordinate (assuming that all other variables are kept constant). Then by Assumption 4 on the rescaling of the mutation kernel, 
and as $\phi \in \mathcal{C}_{b}^{1}\left(\mathbb{R}_{+}^{\left(\begin{array}{c}n \\ 2\end{array}\right)}\right)$,

$(4.38)$

$T_{1.3}$

$$
\begin{aligned}
& =p \int_{X \times K} \bar{\mu}\left(\mathrm{d}\left(x_{2}, \kappa_{2}\right)\right) \beta\left(\kappa_{2}\right) \int_{K} N\left(\alpha_{N}\left(\kappa_{2}, \mathrm{~d} \tilde{\kappa}_{2}\right)-\delta\left(\kappa_{2}, \mathrm{~d} \tilde{\kappa}_{2}\right)\right) \\
& \cdot\left\{\int_{(X \uplus\{z\} \times K)^{n}} \sum_{l=1}^{n} \bar{\mu}^{\otimes(l-1)} \otimes \delta_{\left(x_{2}, \tilde{\kappa}_{2}\right)} \otimes \bar{\mu}^{\otimes(n-l)}(\mathrm{d}(\underline{x}, \underline{\kappa})) \phi\left(\underline{\underline{r}}^{\left(x_{2}, z\right), \frac{1}{N}}(\underline{x})\right) \cdot f(\underline{\kappa})+\mathcal{O}\left((N m(\chi))^{-1}\right)\right\} \\
& +p \cdot \int_{X \times K} \bar{\mu}\left(\mathrm{d}\left(x_{2}, \kappa_{2}\right)\right) \beta\left(\kappa_{2}\right) \int_{K} \alpha_{N}\left(\kappa_{2}, \mathrm{~d} \tilde{\kappa}_{2}\right) \\
& \cdot\left\{\int_{(X \uplus\{z\} \times K)^{n}} \sum_{l=1}^{n} \bar{\mu}^{\otimes(l-1)} \otimes\left(\delta_{\left(z, \tilde{\kappa}_{2}\right)}-\delta_{\left(x_{2}, \tilde{\kappa}_{2}\right)}\right) \otimes \bar{\mu}^{\otimes(n-l)}(\mathrm{d}(\underline{x}, \underline{\kappa})) \frac{\phi\left(\underline{\underline{r}}^{\left(x_{2}, z\right), \frac{1}{N}}(\underline{x})\right)}{\frac{1}{N}} f(\underline{\kappa})+\mathcal{O}\left((N m(\chi))^{-1}\right)\right\} \\
& =p \cdot \int_{(X \times K)^{n}} \bar{\mu}^{\otimes n}(\mathrm{~d}(\underline{x}, \underline{\kappa})) \phi(\underline{\underline{r}}(\underline{x})) \cdot \sum_{l=1}^{n} \beta\left(\kappa_{l}\right) \cdot A^{(l)} f(\underline{\kappa})+o_{N}(1)+\mathcal{O}\left((N m(\chi))^{-1}\right) \\
& +p \cdot \int_{(X \times K)^{n}} \bar{\mu}^{\otimes n}(\mathrm{~d}(\underline{x}, \underline{\kappa})) \sum_{1 \leq l_{1}<l_{2} \leq n}\left\{\beta\left(\kappa_{l_{1}}\right)+\beta\left(\kappa_{l_{2}}\right)\right\} \cdot \frac{\partial \phi}{\partial r_{l_{1}, l_{2}}}(\underline{\underline{r}}(\underline{x})) \cdot f(\underline{\kappa}) \\
& =\Omega_{\text {trait mutation }}^{p, \beta, A} F^{1,(n, \phi, f)}(\chi)+\Omega_{\text {growth }}^{p, \beta} F^{1,(n, \phi, f)}(\chi)+o_{N}(1)+\mathcal{O}\left(\frac{1}{N m(\chi)}\right) .
\end{aligned}
$$

Thus for some $C \in(0, \infty)$,

$$
\begin{aligned}
& \sup _{\chi \in \mathbb{M}_{N}^{K} ; \frac{2}{N} \leq m(\chi) \leq \frac{a_{N}}{N}}\left|g(m(\chi)) \cdot T_{1.3}-\left(\Omega_{\text {trait mutation }}^{p, \beta, A}+\Omega_{\text {growth }}^{p, \beta}\right) F^{g,(n, \phi, f)}(\chi)\right| \\
& \leq C \cdot \sup _{m \in\left[\frac{2}{N}, \frac{a_{N}}{N}\right]}|g(m)| \underset{N \rightarrow \infty}{\longrightarrow} 0,
\end{aligned}
$$

as $g(0)=0$. Moreover, for some sequence $c_{N} \downarrow 0$ for $N \rightarrow \infty$,

$$
\begin{aligned}
& \sup _{\chi \in \mathbb{M}_{N}^{K} ; m(\chi) \geq \frac{a_{N}}{N}}\left|g(m(\chi)) \cdot T_{1.3}-\left(\Omega_{\text {trait mutation }}^{p, \beta,}+\Omega_{\text {growth }}^{p, \beta}\right) F^{g,(n, \phi, f)}(\chi)\right| \\
& \leq C \cdot\left(c_{N}+a_{N}^{-1}\right) \cdot \sup _{m \in\left[\frac{a_{N}}{N}, \infty\right)}|g(m)| \underset{N \rightarrow \infty}{\longrightarrow} 0,
\end{aligned}
$$

as $g$ is bounded and $a_{N} \underset{N \rightarrow \infty}{\longrightarrow} \infty$.

Step 1.4 (The term $E_{1.4}$ ) It turns out that this last term is negligible in the limit as $N \rightarrow \infty$. Indeed, analogous calculations to Step 1.3 show that $E_{1,4}$ goes to zero as $N \rightarrow \infty$ uniformly over all $m \geq \frac{2}{N}$.

Step 2 (The term $T_{2}$ ) Recall $a, b, A, B, C, D$ and $E$ from (4.14), and $T_{2}=a \int \mathrm{d} \bar{\mu} A(D-$ $E)+a \int \mathrm{d} \bar{\mu}(B-C) E+b \cdot \int \mathrm{d} \bar{\mu} A(D+E)$ from (4.18). This term describes the evolution of the total mass. Recall $\Gamma\left(m(\chi), r_{1,2}, \kappa_{1}, \kappa_{2}\right)$ from (2.35) and $\widehat{\Gamma}(m(\chi))$ from (2.40), and $\Omega_{\text {total mass }}^{\beta, \Gamma}:=$ $\Omega_{\text {competition }}^{\Gamma}+\Omega_{\text {mass flow }}^{\beta}+\Omega_{\text {total mass fluctuation }}^{\beta}$ from (2.41). 
Abbreviate

$$
\begin{aligned}
\Gamma\left(m(\chi), r\left(\bar{\mu}, x_{2}\right), \kappa(\bar{\mu}), \kappa_{2}\right) & :=\int \bar{\mu}\left(\mathrm{d}\left(x_{1}, \kappa_{1}\right)\right) \Gamma\left(m(\chi), r\left(x_{1}, x_{2}\right), \kappa_{1}, \kappa_{2}\right) \\
& =\frac{1}{N m(\chi)}(B-C) .
\end{aligned}
$$

Then by (4.20),

$$
\begin{aligned}
& a \int \mathrm{d} \bar{\mu}(B-C) E \\
& =g^{\prime}(m(\chi)) \cdot m(\chi) \cdot \int_{X \times K} \bar{\mu}\left(\mathrm{d}\left(x_{2}, \kappa_{2}\right)\right) \Gamma\left(m(\chi), r\left(\bar{\mu}, x_{2}\right), \kappa(\bar{\mu}), \kappa_{2}\right) \\
& \quad \cdot \int_{(X \times K)^{n}}\left(\frac{N m(\chi) \cdot \bar{\mu}-\delta_{\left(x_{2}, \kappa_{2}\right)}}{N m(\chi)-1}\right)^{\otimes n}(\mathrm{~d}(\underline{x}, \underline{\kappa})) \phi(\underline{\underline{r}}(\underline{x})) \cdot f(\underline{\kappa}) \\
& =g^{\prime}(m(\chi)) \cdot m(\chi) \cdot \hat{\Gamma}(m(\chi)) \cdot \int_{(X \times K)^{n}} \bar{\mu}^{\otimes n}(\mathrm{~d}(\underline{x}, \underline{\kappa})) \phi(\underline{\underline{r}}(\underline{x})) \cdot f(\underline{\kappa}) \\
& \quad+g^{\prime}(m(\chi)) m(\chi)(1 \vee \tilde{\gamma}(m(\chi))) \mathcal{O}\left((N m(\chi))^{-1}\right) \\
& =\Omega_{\text {competition }}^{\Gamma} F^{g,(n, \phi, f)}(\chi)+\mathcal{O}\left((N m(\chi))^{-1}\right),
\end{aligned}
$$

as we have assumed that $m \tilde{\gamma}(m) g^{\prime}(m)$ is bounded.

Further by (4.23),

$$
\begin{aligned}
= & a \int \mathrm{d} \bar{\mu} A(D-E) \\
= & g^{\prime}(m(\chi)) \cdot p \int_{X \times K} \bar{\mu}\left(\mathrm{d}\left(x_{2}, \kappa_{2}\right)\right) \beta\left(\kappa_{2}\right) \int_{K} \alpha_{N}\left(\kappa_{2}, \mathrm{~d} \tilde{\kappa}_{2}\right) \\
& \cdot \int_{(X \uplus\{z\} \times K)^{n}} N m(\chi) \cdot\left(\left(\frac{N m(\chi) \bar{\mu}+\delta_{\left(z, \tilde{\kappa}_{2}\right)}}{N m(x)+1}\right)^{\otimes n}-\left(\frac{N m(\chi) \bar{\mu}-\delta_{\left(x_{2}, \kappa_{2}\right)}}{N m(\chi)-1}\right)^{\otimes n}\right)(\mathrm{d}(\underline{x}, \underline{\kappa})) \phi\left(\underline{\underline{r}}^{\left(x_{2}, z\right), \frac{1}{N}}(\underline{x})\right) \cdot f(\underline{\kappa}) \\
& +g^{\prime}(m(\chi)) \cdot(1-p) \int_{X \times K} \bar{\mu}\left(\mathrm{d}\left(x_{2}, \kappa_{2}\right)\right) \beta\left(\kappa_{2}\right) \\
& \cdot \int_{(X \times K)^{n}} N m(\chi) \cdot\left(\left(\frac{N m(\chi) \bar{\mu}+\delta_{\left(x_{2}, \kappa_{2}\right)}}{N m(\chi)+1}\right)^{\otimes n}-\left(\frac{N m(\chi) \bar{\mu}-\delta_{\left(x_{2}, \kappa_{2}\right)}}{N m(\chi)-1}\right)^{\otimes n}\right)(\mathrm{d}(\underline{x}, \underline{\kappa})) \phi(\underline{\underline{r}}(\underline{x})) \cdot f(\underline{\kappa}) \\
= & 2 g^{\prime}(m(\chi)) \int_{X \times K} \bar{\mu}\left(\mathrm{d}\left(x_{2}, \kappa_{2}\right)\right) \beta\left(\kappa_{2}\right) \int_{(X \times K)^{n}} \sum_{l=1}^{n} \bar{\mu}^{\otimes(l-1)} \otimes\left(\delta_{\left(x_{2}, \kappa_{2}\right)}-\bar{\mu}\right) \otimes \bar{\mu}^{n-l}(\mathrm{~d}(\underline{x}, \underline{\kappa})) \phi(\underline{\underline{r}}(\underline{x})) \cdot f(\underline{\kappa}) \\
& +g^{\prime}(m(\chi)) o_{N}(1)+g^{\prime}(m(\chi)) \mathcal{O}\left((N m(\chi))^{-1}\right) \\
= & 2 g^{\prime}(m(\chi)) \int_{(X \times K)^{n}} \bar{\mu}^{\otimes n}(\mathrm{~d}(\underline{x}, \underline{\kappa})) \sum_{l=1}^{n}\left(\beta\left(\kappa_{l}\right)-\hat{\beta}^{\chi}\right) \phi(\underline{\underline{r}}(\underline{x})) \cdot f(\underline{\kappa})+o_{N}(1)+\mathcal{O}\left((N m(\chi))^{-1}\right) \\
= & \Omega_{\text {mass flow }}^{\beta} F^{g,(n, \phi, f)}(\chi)+o_{N}(1)+\mathcal{O}\left((N m(\chi))^{-1}\right),
\end{aligned}
$$

as we have assumed $g$ is bounded differentiable. 
Moreover,

$$
\begin{aligned}
= & b \int \mathrm{d} \bar{\mu} A(D+E) \\
= & \frac{1}{2} g^{\prime \prime}(m(\chi)) \cdot m(\chi) \cdot \int_{X \times K} \bar{\mu}\left(\mathrm{d}\left(x_{2}, \kappa_{2}\right)\right) \beta\left(\kappa_{2}\right) \int_{K} \alpha_{N}\left(\kappa_{2}, \mathrm{~d} \tilde{\kappa}_{2}\right) \\
& \cdot\left\{p \int_{(X \uplus\{z\} \times K)^{n}}\left(\left(\frac{N m(\chi) \bar{\mu}+\delta_{\left(z, \tilde{\kappa}_{2}\right)}}{N m(x)+1}\right)^{\otimes n}+\left(\frac{N m(\chi) \bar{\mu}-\delta_{\left(x_{2}, \kappa_{2}\right)}}{N m(\chi)-1}\right)^{\otimes n}\right)(\mathrm{d}(\underline{x}, \underline{\kappa})) \phi\left(\underline{\underline{r}}^{\left(x_{2}, z\right), \frac{1}{N}}(\underline{x})\right) \cdot f(\underline{\kappa})\right. \\
& \left.+(1-p) \int_{(X \times K)^{n}}\left(\left(\frac{N m(\chi) \bar{\mu}+\delta_{\left(x_{2}, \kappa_{2}\right)}}{N m(\chi)+1}\right)^{\otimes n}+\left(\frac{N m(\chi) \bar{\mu}-\delta_{\left(x_{2}, \kappa_{2}\right)}}{N m(\chi)-1}\right)^{\otimes n}\right)(\mathrm{d}(\underline{x}, \underline{\kappa})) \phi(\underline{\underline{r}}(\underline{x})) \cdot f(\underline{\kappa})\right\} \\
= & g^{\prime \prime}(m(\chi)) \cdot m(\chi) \cdot \hat{\beta}^{x} \int_{(X \times K)^{n}} \bar{\mu}^{\otimes n}(\mathrm{~d}(\underline{x}, \underline{\kappa})) \phi(\underline{\underline{r}}(\underline{x})) \cdot f(\underline{\kappa})+g^{\prime \prime}(m(\chi)) \cdot m(\chi) \cdot o_{N}(1) \\
= & \Omega_{\text {total mass fluctuation }}^{\beta} F^{g,(n, \phi, f)}(\chi)+o_{N}(1),
\end{aligned}
$$

as we have assumed that $m g^{\prime \prime}(m)$ is bounded.

Repeating the same arguments as in (4.30) and (4.31), (4.34) and (4.35), as well as (4.39) and (4.40) we can conclude once more that

$$
\sup _{\chi \in \mathbb{M}_{N}^{K}}\left|T_{2}-\Omega_{\text {total mass }}^{\beta, \Gamma} F^{g,(n, \phi, f)}(\chi)\right| \underset{N \rightarrow \infty}{\longrightarrow} 0 .
$$

Step 3 (The error-term $E_{3}$ ) Recall $E_{3}$ from (4.18) and $C_{g}$ from (4.11). It turns out that the term $E_{3}$ is negligible in the limit as $N \rightarrow \infty$. Indeed, by (4.23) and Assumption 4

$$
\begin{aligned}
E_{3} & =g^{\prime}(m(\chi)) \cdot \bar{\gamma}_{b} \cdot \mathcal{O}\left(\frac{1}{N}\right)+\mathcal{O}\left(\frac{1}{N}\right) g^{\prime \prime}(m(\chi)) m(\chi)\left(\bar{\gamma}_{b}+\tilde{\gamma}(m(\chi))\right) \\
& +m(\chi) \cdot C_{g}(m(\chi), N) \cdot\left(1 \vee \frac{\tilde{\gamma}(m(\chi))}{N}\right) \cdot \mathcal{O}\left(\frac{1}{N}\right) .
\end{aligned}
$$

Hence $E_{3}$ goes to zero as $N \rightarrow \infty$ uniformly over all $m \geq \frac{2}{N}$.

\section{The COMPACT CONTAINMENT CONDITION}

Let for each $N \in \mathbb{N}, \mathcal{X}^{N}$ be the tree-valued $\left(\ell_{N}, \zeta_{N}, \alpha_{N}\right)$-trait-dependent branching dynamics with mutation and competition rescaled as given in Subsection 2.3. In this section we verify the following compact containment condition.

Proposition 5.1 (Compact containment). Assume that $\left\{\mathcal{X}_{0}^{N} ; N \in \mathbb{N}\right\}$ is a tight family of random elements in $\mathbb{M}^{K}$. Suppose Assumptions 1, 2, 4. (5 and [6 hold as well as (3.3) with $q=1$. Then for every $\epsilon, T>0$ there exists a compact $\mathcal{K}_{\epsilon, T} \subset \mathbb{M}^{K}$ such that

$$
\inf _{N \in \mathbb{N}} \mathbb{P}\left(\mathcal{X}_{t}^{N} \in \mathcal{K}_{T, \epsilon} \text { for all } t \in[0, T]\right) \geq 1-\epsilon .
$$

Recall from Proposition 2.4 the characterization of (pre-)compact sets in $\mathbb{M}^{K}$. To prove the claim of this proposition it is therefore enough to show the following. 
Lemma 5.2 (Compact containment modified). Under the assumptions of Proposition [5.1, for $T, \epsilon_{0}>0$ arbitrarily fixed, for all $k \in \mathbb{N}$ there exist sets $\mathcal{K}_{T, \epsilon_{0}, k} \subset \mathbb{M}^{K}$ such that conditions (i)-(iii) of Proposition 2.4 with $\epsilon=2^{-k}$ are satisfied for all $\chi \in \mathcal{K}_{T, \epsilon_{0}, k}$ and

$$
\inf _{N \in \mathbb{N}} \mathbb{P}\left(\left\{\mathcal{X}_{t}^{N} \in \mathcal{K}_{T, \epsilon_{0}, k} \text { for all } t \in[0, T]\right\}\right) \geq 1-\epsilon_{0} 2^{-k} .
$$

Indeed, set $\mathcal{K}_{T, \epsilon_{0}}:=\bigcap_{k \in \mathbb{N}} \mathcal{K}_{T, \epsilon_{0}, k}$. Then the latter is relatively compact by the characterization of relatively compact sets in Proposition 2.4 and its closure satisfies (5.1).

From now on assume that the assumptions of Proposition 5.1 hold. Let $T>0$ be arbitrarily fixed. We proceed to verify the compact containment condition from Lemma 5.2 in three steps. Let $\epsilon_{0}>0$ be arbitrarily fixed. Item (i) of Proposition 2.4 (control of total mass) is established first. Items (ii) and (iii-a)-(iii-b) (control of traits and the diameter) follow in Step 2. The proof of Item (iii-c) (coverage number by balls of radius $3 \epsilon$ ) is the most involved and concludes the proof in a third step. As a preparation we will start to provide a comparison argument which will allow for reductions to technically simpler situations.

Lemma 5.3 (Comparison). Suppose that $\left(\beta, \gamma_{1}^{\text {birth }}, \gamma_{1}^{\text {death }}\right)$ and $\left(\beta, \gamma_{2}^{\text {birth }}, \gamma_{2}^{\text {death }}\right)$ satisfy the assumptions of Proposition [5.1, $\gamma_{2}^{\text {birth }}\left(m, r, \kappa_{1}, \kappa_{2}\right)=\gamma_{2}^{\text {birth }}\left(\kappa_{2}\right), \gamma_{2}^{\text {death }}\left(m, r, \kappa_{1}, \kappa_{2}\right)=\gamma_{2}^{\text {death }}\left(\kappa_{2}\right)$ and suppose that $\gamma_{1}^{\text {birth }} \leq \gamma_{2}^{\text {birth }}$ and that $\gamma_{2}^{\text {death }} \leq \gamma_{1}^{\text {death }}$. Assume that Lemma 5.2 holds with $\left(\beta, \gamma_{2}^{\text {birth }}, \gamma_{2}^{\text {death }}\right)$. Then it also holds with $\left(\beta, \gamma_{1}^{\text {birth }}, \gamma_{1}^{\text {death }}\right)$.

Proof. Fix $p \in[0,1]$, and couple for each $N \in \mathbb{N}, \mathcal{X}^{N, 1}$ with competition rate $\gamma_{1}^{\text {death }}$ and birthenhancement rate $\gamma_{1}^{\text {birth }}$ to $\mathcal{X}^{N, 2}$ with competition rate $\gamma_{2}^{\text {death }}$ and birth-enhancement rate $\gamma_{2}^{\text {birth }}$ both starting in the same state in such a way that for all realizations and all $t \geq 0, X_{t}^{N, 1} \subseteq X_{t}^{N, 2}$, $r_{t}^{N, 1}=\left.r_{t}^{N, 2}\right|_{X_{t}^{N, 1}}$ and $\mu_{t}^{N, 1} \leq \mu_{t}^{N, 2}$. Such a coupling is always possible as $\mathcal{X}^{N, 2}$ can only result in additional births or omissions of deaths that are captured by means of $\mu_{t}^{N, 2}-\mu_{t}^{N, 1}$ and have no impact on the death- respectively birth-rates in (2.18) respectively (2.21).

Recall that by Remark 2.9 there exists a finite constant $C>0$ such that $\gamma^{\text {birth }}\left(m, r, \kappa_{1}, \kappa_{2}\right) \leq$ $C \beta\left(\kappa_{2}\right)$ for all $m \geq 0, r \geq 0, \kappa_{1}, \kappa_{2} \in K$. For the remainder of this section, assume that without loss of generality for $m \geq 0, r \geq 0$ and $\kappa, \kappa^{\prime} \in K$,

$$
\gamma^{\text {death }}\left(m, r, k, k^{\prime}\right) \equiv 0 \text { and } \gamma^{\text {birth }}\left(m, r, k, k^{\prime}\right) \equiv C \beta\left(\kappa^{\prime}\right) .
$$

In particular, the model now satisfies the additional assumptions of Proposition 3.1(iii).

Step 1: The mass [Item (i) of Proposition 2.4]. For all $T, \epsilon_{0}>0, k \in \mathbb{N}$ there exists $M_{k}>0$ big enough such that

$$
\sup _{N \in \mathbb{N}} \mathbb{P}\left(\sup _{t \in[0, T]} m\left(\chi_{t}^{N}\right)>M_{k}\right)<\frac{1}{3} \epsilon_{0} 2^{-k} .
$$

This follows from (3.4) and Markov's inequality.

Remark 5.4 (Excluding empty clans). Recall the remarks in and around (2.17)-(2.24) with regards to empty clans, that is, clans $x \in X$ that satisfy $n_{x}=0=\mu(\{x\} \times K)$. For the remainder of this section, we assume without loss of generality that every $x \in X_{t}^{N}$ satisfies $\mu(\{x\} \times K)>0$. Indeed, it remains to verify Items (ii) and (iii) of Proposition 2.4. Empty clans are without effect on Item (ii) and can be included into the exceptional sets $X_{\epsilon}^{c}$ in Item (iii). 
For $(X, r, \mu) \in \mathbb{M}^{K}$ let

$$
\mathcal{S}_{\epsilon}(\chi):=\min \{\# \text { balls of radius } \epsilon \text { needed to cover } \operatorname{supp}(\mu(\cdot \times K))\} .
$$

As we assume that $\left\{\mathcal{X}_{0}^{N} ; N \in \mathbb{N}\right\}$ is a tight family of random elements in $\mathbb{M}^{K}$, Proposition 3.1(iii) yields for each $\epsilon_{0}>0$ and $k \in \mathbb{N}$ the existence of $m_{k}=m_{k}\left(\epsilon_{0}\right)>0$ such that

$$
\sup _{n \in \mathbb{N}} \mathbb{P}\left(\sup _{t \in[0, T]} m\left(\mathcal{X}_{t}^{N}\right)>2^{-k} / 4 \mid m\left(\mathcal{X}_{0}^{N}\right) \leq m_{k}\right) \leq \frac{1}{24} \epsilon_{0} 2^{-k} .
$$

Moreover, Proposition 2.4 yields the existence of $L_{k}=L_{k}\left(\epsilon_{0}\right), N_{k}=N_{k}\left(\epsilon_{0}\right)>0$ big enough such that

$$
\begin{aligned}
& \inf _{N \in \mathbb{N}} \mathbb{P}\left(\exists\left(X_{0}^{N}\right)_{k} \subseteq X_{0}^{N}: \mu_{0}^{N}\left(\left(X_{0}^{N}\right)_{k}^{c} \times K\right) \leq m_{k}, \operatorname{diam}\left(\left(X_{0}^{N}\right)_{k}\right) \leq \frac{L_{k}}{2} \text { and } \mathcal{S}_{2^{-k}}\left(\left(x_{0}^{N}\right)_{k}\right) \leq \frac{N_{k}}{2}\right) \\
& >1-\frac{1}{24} \epsilon_{0} 2^{-k} .
\end{aligned}
$$

Step 2: The trait and the diameter [Item (ii), (iii-a,b) of Proposition 2.4. In this step we prove the following (recall that we consider $\epsilon=2^{-k}$ in (i)-(iii) of Proposition 2.4):

Lemma 5.5. For all $k \in \mathbb{N}$ there exists $K_{k} \subseteq K$ compact and $L_{k}>0$ big enough such that

$$
\inf _{N \in \mathbb{N}} \mathbb{P}_{N}\left(\sup _{t \in[0, T]} \mu_{t}^{N}\left(X_{t}^{N} \times K_{k}^{c}\right) \leq 2^{-k}\right)>1-\frac{1}{6} \epsilon_{0} 2^{-k}
$$

and

$$
\begin{aligned}
\inf _{N \in \mathbb{N}} \mathbb{P}\left(\forall t \in[0, T], \exists\left(X_{t}^{N}\right)_{k} \subseteq X_{t}^{N}:\right. & \sup _{t \in[0, T]} \mu_{t}^{N}\left(\left(\left(X_{t}^{N}\right)_{k}\right)^{c} \times K\right) \leq 2^{-k} / 2, \\
& \left.\sup _{t \in[0, T]} \operatorname{diam}\left(\left(X_{t}^{N}\right)_{k}\right) \leq L_{k}\right)>1-\frac{1}{6} \epsilon_{0} 2^{-k} .
\end{aligned}
$$

Proof of Lemma 5.5. Consider the individual-based description of our trait-dependent branching particle model with mutation and competition given in Subsection 2.2. Notice that due to the branching mechanism, each particle $x$ alive at time $t \geq 0$ is the descendant of a well-defined ancestor, $A_{t-s}(x)$, at time $0 \leq s \leq t$. If $A_{t}(x)=A_{t}(y)$, let $T(x, y):=\sup \left\{s \in(0, t]: A_{t-s}(x)=\right.$ $\left.A_{t-s}(y)\right\}$ denote the time instant at which the ancestral lines of these particles had split. Extend this definition to letting $T(x, y):=0$ in case that $A_{t}(x) \neq A_{t}(y)$. If we define the (genetic) age $a_{t}^{N}(\{x\})$ of an individual $x$ living at time $t$ as $\zeta_{N}\left(=\frac{1}{N}\right)$ times the number of mutations which separate $A_{t}(x)$ and $x$, then the genetic distance of particles $x$ and $y$ satisfies

$$
\begin{aligned}
& r_{t}^{N}(x, y) \\
& =a_{t}^{N}(\{x\})-a_{T(x, y)}^{N}\left(\left\{A_{(t-T(x, y))}(x)\right\}\right)+a_{t}^{N}(\{y\})-a_{T(x, y)}^{N}\left(\left\{A_{(t-T(x, y))}(y)\right\}\right)+r_{0}^{N}\left(A_{t}(x), A_{t}(y)\right) \\
& \leq a_{t}^{N}(\{x\})+a_{t}^{N}(\{y\})+r_{0}^{N}\left(A_{t}(x), A_{t}(y)\right) .
\end{aligned}
$$

Fix $k \in \mathbb{N}$. Recall the notation $n_{x}$ for the number of clones in a clan $x$ from (2.14). We consider the following two auxiliary measure-valued processes $\chi, \eta$ which are Markov jump processes taking values in the space of finite measures on $K$ respectively $K \times \mathbb{R}_{+}$and are given for each $t \geq 0$ by

$$
\chi_{t}^{N}:=\frac{1}{N} \sum_{x \in X_{t}^{N}} n_{x} \delta_{\kappa_{t}(x)} \quad \text { and } \quad \eta_{t}^{N}:=\frac{1}{N} \sum_{x \in X_{t}^{N} ; A_{t}(x) \in\left(X_{0}^{N}\right)_{k}} n_{x} \delta_{\left(\kappa_{t}(x), a_{t}^{N}(\{x\})\right)} .
$$


with $\left(X_{0}^{N}\right)_{k}$ as in (5.6).

As by Assumption (5.2) none of the rates depend on the genetic distance, we obtain the following:

Lemma 5.6. Assume that the assumptions of Proposition 5.1 hold. If the families $\left\{\chi^{N} ; N \in \mathbb{N}\right\}$ and $\left\{\eta^{N} ; N \in \mathbb{N}\right\}$ satisfy respective compact containment conditions, then the statement of Lemma 5.5 follows.

Proof of Lemma 5.6. Fix $T>0$, and $\epsilon_{0}>0$. By the tightness assumptions and EK86, Theorem 3.6.3 and Remark 3.6.4], for any $k \in \mathbb{N}$, we can find a compact subset $K_{k} \subseteq K$ and a number $L_{k}>0$ such that

$$
\sup _{N \in \mathbb{N}} \mathbb{P}\left(\sup _{t \in[0, T]} \chi_{t}^{N}\left(K_{k}^{c}\right)>2^{-k}\right) \leq \frac{1}{12} \epsilon_{0} 2^{-k} .
$$

and

$$
\sup _{N \in \mathbb{N}} \mathbb{P}\left(\sup _{t \in[0, T]} \eta_{t}^{N}\left(\left(K_{k} \times\left[0, \frac{L_{k}}{4}\right]\right)^{c}\right)>2^{-k} / 2\right) \leq \frac{1}{12} \epsilon_{0} 2^{-k} .
$$

By (5.11) and the definition of $\chi$,

$$
\sup _{N \in \mathbb{N}} \mathbb{P}\left(\sup _{t \in[0, T]} \mu_{t}^{N}\left(X_{t}^{N} \times K_{k}^{c}\right)>2^{-k}\right) \leq \frac{1}{12} \epsilon_{0} 2^{-k}
$$

and (5.7) follows.

Next set for each $t \in[0, T]$,

$$
\left(X_{t}^{N}\right)_{k}:=\left\{x \in X_{t}^{N}: A_{t}(x) \in\left(X_{0}^{N}\right)_{k} \text { and } a_{t}^{N}(\{x\}) \leq L_{k} / 4\right\} .
$$

Then $\operatorname{diam}\left(\left(X_{t}^{N}\right)_{k}\right) \leq L_{k}$ and by (5.5), (5.6), (5.12) and the definition of $\eta$,

$$
\begin{aligned}
& \sup _{N \in \mathbb{N}} \mathbb{P}\left(\sup _{t \in[0, T]} \mu_{t}^{N}\left(\left(X_{t}^{N}\right)_{k}^{c} \times K\right)>2^{-k} / 2 \text { or } \sup _{t \in[0, T]} \operatorname{diam}\left(\left(X_{t}^{N}\right)_{k}\right)>L_{k}\right) \\
& \leq \frac{1}{24} \epsilon_{0} 2^{-k}+\frac{1}{24} \epsilon_{0} 2^{-k}+\sup _{N \in \mathbb{N}} \mathbb{P}\left(\sup _{t \in[0, T]} \eta_{t}^{N}\left(\left(K_{k} \times\left[0, \frac{L_{k}}{2}\right]\right)^{c}\right)>2^{-k} / 2\right) \\
& \leq \frac{1}{6} \epsilon_{0} 2^{-k},
\end{aligned}
$$

and the second claim (5.8) follows.

To finish the proof of Lemma 5.5, we need to verify the compact containment for the measurevalued processes. Recall (5.2). In this particular setting the claim is covered by Kli14, Theorem 3.4] (applied to $r(t, y):=\beta\left(\pi_{K}\left(y_{t}\right)\right)$ with $\pi_{K}: K \times \mathbb{R}_{+} \rightarrow K$ denoting the projection map on to the trait, $b(t, y):=C \beta\left(\pi_{K}\left(y_{t}\right)\right), D(t, y) \equiv 0, U\left(t, y, y^{\prime}\right) \equiv 0$ and $\alpha_{N}\left((\kappa, a), \mathrm{d}\left(\kappa^{\prime}, a^{\prime}\right)\right):=$ $\alpha_{N}\left(\kappa, \mathrm{d} \kappa^{\prime}\right) \delta_{a+\frac{1}{N} \mathbf{1}\left\{\kappa \neq \kappa^{\prime}\right\}}\left(d a^{\prime}\right)$. Here the left hand sides refer to the set-up used in Kli14] and the right hand sides to our set-up.).

Remark 5.7 (Non-ultrametric setup). In the present article and Kli14, because of the use of exponential times in the modelling of birth- and death-events, the analysis of the modulus of continuity of the trait-history of a particle (cf. [Kli14, Definition 5.3]) plays a major role in obtaining appropriate bounds. In the present article, the need for such an analysis arises due to the non-ultrametric setup, where instead of defining genetic distance to be twice the time to the most recent common ancestor (MRCA) (cf., for example, GPW13, (2.27)] in the 
context of tree-valued Moran dynamics), genetic distance is taken to be the sum of the number of mutations of two individuals back in time to their MRCA. The control of the modulus of continuity along the path of an individual now allows us to relate genetic distance to time by considering age as part of the type-space in Kli14.

Step 3: Coverage number of balls of radius $3 \epsilon$ [Item (iii-c) of Proposition 2.4]. In this step we show the following.

Lemma 5.8. For all $k \in \mathbb{N}$ there exists $N_{k}>0$ such that

$$
\begin{gathered}
\inf _{N \in \mathbb{N}} \mathbb{P}\left(\forall t \in[0, T], \exists\left(X_{t}^{N}\right)_{k}^{\prime} \subseteq X_{t}^{N}: \sup _{t \in[0, T]} \mu_{t}^{N}\left(\left(\left(X_{t}^{N}\right)_{k}^{\prime}\right)^{c} \times K\right)<2^{-k} / 2,\right. \\
\left.\sup _{t \in[0, T]} \mathcal{S}_{3 \cdot 2^{-k}}\left(\left(x_{t}^{N}\right)_{k}^{\prime}\right)<N_{k}\right)>1-\frac{1}{3} \epsilon_{0} 2^{-k}
\end{gathered}
$$

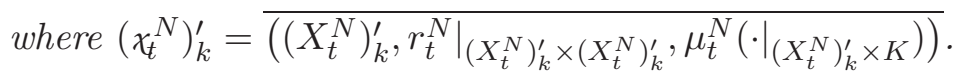

Proof. Fix $k \in \mathbb{N}$. Choose $m_{k},\left(X_{0}^{N}\right)_{k}$ and $N_{k}$ according to (5.5) and (5.6).

We first argue that there exists $t_{0}=t_{0}\left(\epsilon_{0}, k\right)>0$ small enough such that for all $0 \leq t \leq t_{0}$ the genetic distances of a large enough proportion of particles alive at time $t$ to their ancestors at time 0 are at most $\epsilon=2^{-k}$ with high probability, that is, such that

$$
\begin{gathered}
\inf _{N \in \mathbb{N}} \mathbb{P}\left(\forall t \in\left[0, t_{0}\right], \exists\left(\tilde{X}_{t}^{N}\right)_{k} \subseteq X_{t}^{N}: \sup _{t \in\left[0, t_{0}\right]} \mu_{t}^{N}\left(\left(\left(\tilde{X}_{t}^{N}\right)_{k}\right)^{c} \times K\right)<2^{-k} / 4,\right. \\
\left.\sup _{t \in\left[0, t_{0}\right]} \max _{x \in \tilde{X}_{t}^{N}} a_{t}^{N}(\{x\})<2^{-k}\right)>1-\frac{1}{12} \epsilon_{0} 2^{-k} .
\end{gathered}
$$

This follows indeed immediately from [Kli14, Lemma 3.9].

With this choice of $t_{0}$, we can conclude from (5.5) (recall that $\left.m(\chi)=\mu(X \times K)\right)$ and (5.6) by the triangle inequality that

$$
\begin{gathered}
\inf _{N \in \mathbb{N}} \mathbb{P}\left(\forall t \in\left[0, t_{0}\right], \exists\left(\tilde{X}_{t}^{N}\right)_{k} \subseteq X_{t}^{N}: \sup _{t \in\left[0, t_{0}\right]} \mu_{t}^{N}\left(\left(\left(\tilde{X}_{t}^{N}\right)_{k}\right)^{c} \times K\right)<2^{-k} / 2,\right. \\
\left.\sup _{t \in\left[0, t_{0}\right]} \mathcal{S}_{3 \cdot 2^{-k}}\left(\left(\tilde{x}_{t}^{N}\right)_{k}\right) \leq N_{k} / 2\right)>1-\frac{1}{6} \epsilon_{0} 2^{-k} .
\end{gathered}
$$

It then remains to show that

$$
\begin{gathered}
\inf _{N \in \mathbb{N}} \mathbb{P}\left(\forall t \in\left[t_{0}, T\right], \exists\left(\tilde{X}_{t}^{N}\right)_{k} \subseteq X_{t}^{N}: \sup _{t \in\left[t_{0}, T\right]} \mu_{t}^{N}\left(\left(\left(\tilde{X}_{t}^{N}\right)_{k}\right)^{c} \times K\right)<2^{-k} / 2,\right. \\
\left.\sup _{t \in\left[t_{0}, T\right]} \mathcal{S}_{3 \cdot 2^{-k}}\left(\left(\tilde{x}_{t}^{N}\right)_{k}\right) \leq N_{k} / 2\right)>1-\frac{1}{6} \epsilon_{0} 2^{-k} .
\end{gathered}
$$

We will first prove the statement in case the genetic distance $r$ (counting the number of substitutions due to mutation) is replaced by the genealogical distance $\tilde{r}$ (which is twice the time to the most recent ancestor), that is, in contrast to (2.22) distances (between any two distinct individuals) grow deterministically with time at speed 2. In the set-up of genealogical distance our model fits into the class of finite population models (compare, GPW13, Definition 2.18]). Put for each $t \in[0, T]$,

$$
\left(X_{t}^{N}\right)_{k}:=\left\{x \in X_{t}^{N}: A_{t}(x) \in\left(X_{0}^{N}\right)_{k}\right\}
$$


the set of descendants of $\left(X_{0}^{N}\right)_{k}$ at time $t$. It then remains to show the following:

Lemma 5.9. For all $0<\xi \leq t<T$, the family $\left\{\mathcal{S}_{2 \xi}\left(\left(\left(X_{t}^{N}\right)_{k}, \tilde{r}, \mu_{t}^{N}\left(\left.\cdot\right|_{\left(X_{t}^{N}\right)_{k} \times K}\right)\right)\right) ; N \in \mathbb{N}\right\}$ is tight.

Remark 5.10. Assume that $\left\{\mathcal{S}_{2 \xi}\left(\left(\left(X_{t}^{N}\right)_{k}, \tilde{r}, \mu_{t}^{N}\left(\left.\cdot\right|_{\left(X_{t}^{N}\right)_{k} \times K}\right)\right)\right) ; N \in \mathbb{N}\right\}$ is tight for all $0<$ $\xi \leq t<T$, then we can literally copy the proof of [GPW13, Lemma 6.7a)] to obtain for all $0<\xi \leq t<T$, the existence of $C_{\xi}$ such that

$$
\sup _{N \in \mathbb{N}} \mathbb{P}\left(\sup _{t \in[\xi, T)} \mathcal{S}_{2 \xi}\left(\left(\left(X_{t}^{N}\right)_{k}, \tilde{r}, \mu_{t}^{N}\left(\left.\cdot\right|_{\left(X_{t}^{N}\right)_{k} \times K}\right)\right)\right)>C_{\xi}\right) \leq 2 \xi
$$

Proof of Lemma 5.9. Until the end of this proof we set $\left(\tilde{x}_{t}^{N}\right)_{k}:=\overline{\left(\left(X_{t}^{N}\right)_{k}, \tilde{r}, \mu_{t}^{N}\left(\left.\cdot\right|_{\left(X_{t}^{N}\right)_{k} \times K}\right)\right)}$. Fix $\xi \leq t<T$. It is enough to show that for all $\delta>0$ one can find $C=C(\delta)>0$ such that

$$
\sup _{N \in \mathbb{N}} \mathbb{P}\left(\mathcal{S}_{2 \xi}\left(\left(\tilde{x}_{t}^{N}\right)_{k}\right)>C\right)<\delta .
$$

By reasoning as in the derivation of (5.3) we can find a constant $\tilde{M}_{\xi}$ such that

$$
\sup _{N \in \mathbb{N}} \mathbb{P}\left(\sup _{t \in[0, T]} m\left(\left(\tilde{\chi}_{t}^{N}\right)_{k}\right)>\tilde{M}_{\xi}\right)<\delta / 2
$$

holds and thus for $t \in[\xi, T)$ arbitrary, using Markov's inequality, we obtain

$$
\begin{aligned}
\mathbb{P}\left(\mathcal{S}_{2 \xi}\left(\left(\tilde{x}_{t}^{N}\right)_{k}\right)>C\right) & \leq \frac{\delta}{2}+\mathbb{P}\left(\mathcal{S}_{2 \xi}\left(\left(\tilde{x}_{t}^{N}\right)_{k}\right)>C, m\left(\left(\tilde{x}_{t-\xi}^{N}\right)_{k}\right) \leq \tilde{M}_{\xi}\right) \\
& \leq \frac{\delta}{2}+\frac{1}{C} \mathbb{E}\left[\mathcal{S}_{2 \xi}\left(\left(\tilde{x}_{t}^{N}\right)_{k}\right) \mathbf{1}_{\left\{m\left(\left(\tilde{x}_{t-\xi}^{N}\right)_{k}\right) \leq \tilde{M}_{\xi}\right\}}\right] .
\end{aligned}
$$

As we have assumed (5.2), if we see a branching event, the probability that it leads to birth rather than death of an individual does not depend on the individual's type. Moreover, by (2.18) respectively (2.21) the total death- respectively birth-rate of a particle only depends on its clan's trait. As a result, the progenies of different clans evolve independently from each other and we obtain with (2.15),

$$
\begin{aligned}
& \mathbb{E}\left[\mathcal{S}_{2 \xi}\left(\left(\tilde{x}_{t}^{N}\right)_{k}\right) \mathbf{1}_{\left\{m\left(\left(\tilde{x}_{t-\xi}^{N}\right)_{k}\right) \leq \tilde{M}_{\xi}\right\}}\right] \\
& \leq N \tilde{M}_{\xi} \cdot \sup _{\kappa \in K} \mathbb{P}(\text { a particle starting with trait } \kappa \text { has a child present after a period of time } \xi) .
\end{aligned}
$$

We can now assume without loss of generality that $\beta(\cdot) \equiv \beta$ with $\beta>0$ as in Assumption 5. Indeed, we can couple both models such that the birth events and death events match, while the times between two successive branching events are longer for the lower bound $\beta$ than for the type dependent $\beta(\cdot)$. As a result, the progeny of a particle $x$ gets extinct later in the model using $\beta$. Apply the theory of continuous-time birth- and death-processes to the $N^{\text {th }}$-model with birth- respectively death-rates of $N \underline{\beta}$ respectively $N \underline{\beta}(1+C / N)$ to conclude that

$$
\mathbb{E}\left[\mathcal{S}_{2 \xi}\left(\left(\tilde{\chi}_{t}^{N}\right)_{k}\right) \mathbf{1}_{\left\{m\left(\left(\tilde{x}_{t-\xi}^{N}\right)_{k}\right) \leq \tilde{M}_{\xi}\right\}}\right] \leq N \tilde{M}_{\xi} \cdot \text { const. } N^{-1} .
$$

Now choose $C$ big enough in (5.24) to obtain (5.22). 
We next relate time to genetic distance in order to obtain a bound on $\mathcal{S}_{2 \xi}\left(\left(\left(X_{t}^{N}\right)_{k}, r, \mu_{t}^{N}\left(\left.\cdot\right|_{\left(X_{t}^{N}\right)_{k} \times K}\right)\right)\right)$ from the bound on $\mathcal{S}_{2 \xi}\left(\left(\left(X_{t}^{N}\right)_{k}, \tilde{r}, \mu_{t}^{N}\left(\left.\cdot\right|_{\left(X_{t}^{N}\right)_{k} \times K}\right)\right)\right)$. In what follows we estimate the mass of the exceptional subset of $\left(X_{t}^{N}\right)_{k}$ of those individuals whose ancestors at time $t-\xi$ are in genetic distance greater than $\epsilon=2^{-k}$.

Lemma 5.11 (Too fast evolving individuals). For all $0<t_{0}<T, \sigma>0$ and $k \in \mathbb{N}$ there exists $0<s_{0}=s_{0}\left(t_{0}, T, \sigma, k\right)<t_{0} / 2$ small enough such that

$$
\sup _{N \geq 3 \cdot 2^{k}} \mathbb{P}\left(\exists t \in\left[t_{0}, T\right]: \mu_{t}^{N}\left(x \in X_{t}^{N}: a_{t}^{N}(\{x\})-a_{t-s_{0}}^{N}\left(A_{t-s_{0}}(\{x\}, t)\right) \in\left[2^{-k}, \infty\right)\right) \geq 2^{-k} / 4\right) \leq \sigma,
$$

where we denote by $A_{s}(\{x\}, t) \in X_{s}^{N}$ the clan that contains the ancestors at time $0 \leq s \leq t$ of all the individuals in the clan $x \in X_{t}^{N}$ at time $t$.

Proof. This is covered by [Kli14, Lemma 3.9], which gives the analogous result phrased in terms of modulus of continuity of the historical process associated with $\eta^{N}$ from (5.10). As the maximal jump-size in age is $1 / N$, (5.27) follows.

We now combine Lemma 5.11 with Remark 5.10 to finish the proof of Lemma 5.8 ,

1. Case: $N<3 \cdot 2^{k}$.

Recall that $\epsilon=2^{-k}$. Choose $\left(X_{t}^{N}\right)_{k}=X_{t}^{N}$. By reasoning as in (5.3), we can choose $M_{k}^{\prime}>0$ big enough such that

$$
\sup _{N \in \mathbb{N}} \mathbb{P}\left(\sup _{t \in[0, T]} m\left(x_{t}^{N}\right)>M_{k}^{\prime}\right)<\frac{1}{12} \epsilon_{0} 2^{-k} .
$$

For $N<3 \cdot 2^{k}$ the number of individuals alive at time $t$ is $N m\left(x_{t}^{N}\right)$ and thus

$$
\sup _{t \in\left[t_{0}, T\right]} \mathcal{S}_{2^{-k}}\left(x_{t}^{N}\right)<3 \cdot 2^{k} M_{k}^{\prime}
$$

with probability greater or equal to $1-\frac{1}{12} \epsilon_{0} 2^{-k}$. Now (5.19) holds with $N_{k} / 2=3 \cdot 2^{k} M_{k}^{\prime}$. It remains to investigate:

2. Case: $N \geq 3 \cdot 2^{k}$.

Recall the constants $t_{0}=t_{0}(k)$ from (5.17). For $t \in[0, T]$, let

$$
\left(X_{t}^{N}\right)_{k}^{\prime}:=\left(\left(X_{t}^{N}\right)_{k} \backslash\left\{x \in X_{t}^{N}: a_{t}^{N}(\{x\})-a_{t-s_{0}}^{N}\left(A_{t-s_{0}}(\{x\}, t)\right) \in\left[2^{-k}, \infty\right)\right\} .\right.
$$

By Remark 5.10 we obtain for $\xi=s_{0} \wedge \frac{1}{48} \epsilon_{0} 2^{-k}$,

$$
\sup _{N \in \mathbb{N}} \mathbb{P}^{N}\left(\sup _{t \in\left[t_{0}, T\right)} \mathcal{S}_{2 s_{0}}\left(\left(\left(X_{t}^{N}\right)_{k}^{\prime}, \tilde{r}, \mu_{t}^{N}\left(\left.\cdot\right|_{\left(X_{t}^{N}\right)_{k}^{\prime} \times K}\right)\right)\right)>C_{\xi}\right) \leq \frac{1}{24} \epsilon_{0} 2^{-k}
$$

for some constant $C_{\xi}$. An $\epsilon$-coverage with $\epsilon=2^{-k}$ of $\left(X_{t}^{N}\right)_{k}^{\prime}$ for $t \in\left[t_{0}, T\right]$ can now be constructed as follows: Go back $0<s_{0}<t_{0} / 2$ in time, determine the number of ancestors of $\left(X_{t}^{N}\right)_{k}^{\prime}$, which is smaller or equal to $C_{\xi}$ with probability at least $1-\frac{1}{24} \epsilon_{0} 2^{-k}$ by (5.31). Then the genetic distance of the particles in $\left(X_{t}^{N}\right)_{k}^{\prime}$ to their ancestors at time $t-s_{0}$ is indeed bounded by $\epsilon=2^{-k}$ by the definition of $\left(X_{t}^{N}\right)_{k}^{\prime}$ in (5.30). Lemma 5.11 for the choice $\sigma=\frac{1}{24} \epsilon_{0} 2^{-k}$ further yields that (5.19) holds with probability at least $1-\frac{1}{6} \epsilon_{0} 2^{-k}$. We obtain (5.19) with $N_{k} / 2=\max \left\{3 \cdot 2^{k} M_{k}^{\prime}, C_{\xi}\right\}$. 
Completion of the proof of Lemma 5.2 and thereby of Proposition [5.1. Combine (5.3), Lemma 5.5 and Lemma 5.8, replacing $\left(X_{t}^{N}\right)_{k}$ by the intersection of the respective sets and choosing $N_{\epsilon}=\max \left\{M_{k}, L_{K}, N_{k}\right\}$ for $\epsilon=3 \cdot 2^{-k}$ in Proposition 2.4.

\section{Finishing up (Proofs of Theorems 1 and 2)}

Let for each $N \in \mathbb{N}, \mathcal{X}^{N}$ be the tree-valued $\left(\ell_{N}, \zeta_{N}, \alpha_{N}\right)$-trait-dependent branching dynamics with mutation and competition rescaled as given in Subsection 2.3. In this section we want to collect the results from the previous sections to present the proof of tightness (Theorem 1) and the fact that any limit process satisfies the $(\Omega, \mathcal{D}(\Omega)$ )-martingale problem (Theorem 2) with $(\Omega, \mathcal{D}(\Omega))$ as in (2.36) -2.37$)$.

We will apply [EK86, Remark 4.5.2]. This remark states that the sequence $\left(\mathcal{X}^{N}\right)_{N \in \mathbb{N}}$ is relatively compact if

(i) the (strong) compact containment condition (5.1) holds,

(ii) the closure of $\widetilde{\Pi}$ (cf. (4.3) ) contains an algebra which separates points in $\mathbb{M}^{K}$ and vanishes nowhere, and

(iii) for all $F \in \widetilde{\Pi}$, (4.4) holds.

In addition, any limit process satisfies the $(\Omega, \widetilde{\Pi})$-martingale problem.

As (i) is covered by Proposition 5.1 and (iii) is covered by Proposition $4.3\left(\tilde{\gamma}(m)=(1 \vee m) \bar{\gamma}_{d}\right.$ by Assumption [6) it therefore remains to show the following two facts.

Proposition 6.1. The subspace $\widetilde{\Pi} \subseteq \mathcal{D}(\Omega)$ separates points and vanishes nowhere. Moreover, its closure contains an algebra.

Proof. Recall that a class $\chi:=\overline{(X, r, \mu)} \in \mathbb{M}^{K}$ is uniquely characterized by the total mass $m(\chi)$ and the marked distance matrix distribution $\nu^{\chi} \in \mathcal{M}_{1}\left(\mathbb{R}_{+}^{\left(\begin{array}{c}N \\ 2\end{array}\right)} \times K^{\mathbb{N}}\right)$ (cf. (2.6) ). Thus a separating set for $\mathbb{M}^{K}$ are clearly the bounded continuous functions of the form $e^{-\lambda m(\chi)} \int \nu^{\chi}(\mathrm{d}(\underline{\underline{r}}, \underline{\kappa})) \phi(\underline{\underline{r}}) f(\underline{\kappa})$ for some $\left.n \in \mathbb{N}, \lambda \geq 0, \phi \in \mathcal{C}_{b}\left(\mathbb{R}^{(} \begin{array}{l}n \\ 2\end{array}\right)\right)$ and $f \in \mathcal{C}_{b}\left(K^{n}\right)$. As the subspaces $\mathcal{C}_{b}^{1}\left(\mathbb{R}^{\left(\begin{array}{l}n \\ 2\end{array}\right)}\right)$ of $\mathcal{C}_{b}\left(\mathbb{R}^{\left.\left(\begin{array}{l}n \\ 2\end{array}\right)\right)}\right.$ and $\mathcal{D}(A)$ of $\mathcal{C}_{b}(K)$ are dense (also see Assumption 4), the subspace

$$
\begin{aligned}
& \left\{\left(g(m)+e^{-\lambda m(\chi)}\right) \int \nu^{\chi}(\mathrm{d}(\underline{\underline{r}}, \underline{\kappa})) \phi(\underline{\underline{r}}) f(\underline{\kappa}) ; \lambda \geq 0, \epsilon \geq 0, n \in \mathbb{N},\right. \\
& \quad g \in \mathcal{C}_{b}^{3}\left(\mathbb{R}_{+}\right) \text {such that } \operatorname{supp}(g) \subset[0, \epsilon], g^{\prime}(0)=\lambda, \phi \in \mathcal{C}_{b}^{1}\left(\mathbb{R}^{\left(\begin{array}{l}
n \\
2
\end{array}\right)}\right), f \in \mathcal{C}_{b}\left(K^{n}\right) \text { with } \\
& \left.\quad f\left(\kappa_{1}, \ldots, \kappa_{l-1}, \cdot, \kappa_{l+1}, \ldots, \kappa_{n}\right) \in \mathcal{D}(A), \forall l=1, \ldots, n, \forall \kappa_{1}, \ldots, \kappa_{l-1}, \kappa_{l+1}, \ldots, \kappa_{n} \in K\right\} \subset \widetilde{\Pi}
\end{aligned}
$$

also separates points in $\mathbb{M}^{K}$.

As the above subspace of $\widetilde{\Pi}$ contains constants, it vanishes nowhere. Moreover, any function $F \in \Pi^{2,1, A}$ can be approximated by a sequence in $\widetilde{\Pi}$. As $\Pi^{2,1, A}$ is an algebra, the closure of $\widetilde{\Pi}$ clearly contains an algebra.

Proposition 6.2 (Extension of the domain). If $\mathcal{X}$ is a $\mathbb{M}^{K}$-valued process which satisfies the $(\Omega, \widetilde{\Pi})$-martingale problem, then it satisfies the $(\Omega, \mathcal{D}(\Omega))$-martingale problem as well. 
Proof. Fix $F \in \mathcal{D}(\Omega)$. We have seen that we can find a sequence $\left(F_{n}\right)$ in $\widetilde{\Pi}$ such that $F_{n} \rightarrow F$ as $n \rightarrow \infty$ and $\Omega F_{n} \rightarrow \Omega F$, as $n \rightarrow \infty$, boundedly pointwise. Indeed, if this is the case we can conclude from the fact that the process

$$
M^{F_{n}}:=\left(F_{n}\left(\mathcal{X}_{t}\right)-F_{n}\left(\mathcal{X}_{0}\right)-\int_{0}^{t} \Omega F_{n}\left(\mathcal{X}_{s}\right) \mathrm{d} s\right)_{t \geq 0}
$$

is a martingale with respect to the canonical filtration of $\mathcal{X}$, that the process

$$
\begin{aligned}
M^{F} & :=\lim _{n \rightarrow \infty}\left(F_{n}\left(\mathcal{X}_{t}\right)-F_{n}\left(\mathcal{X}_{0}\right)-\int_{0}^{t} \Omega F_{n}\left(\mathcal{X}_{s}\right) \mathrm{d} s\right)_{t \geq 0} \\
& =\left(F\left(\mathcal{X}_{t}\right)-F\left(\mathcal{X}_{0}\right)-\int_{0}^{t} \Omega F\left(\mathcal{X}_{s}\right) \mathrm{d} s\right)_{t \geq 0}
\end{aligned}
$$

is a martingale as well.

AcKnowledgement. We thank Wolfgang Löhr for many fruitful discussions.

\section{REFERENCES}

[BK14] Krzysztof Bartoszek and Michał Krzemiński. Critical case stochastic phylogenetic tree model via the Laplace transform. Demonstratio Math., 47(2):474-481, 2014.

[BvH99] Ellen Baake and Arndt von Haeseler. Distance measures in terms of substitution processes. Theor. Popul. Biol., 55:166-175, 1999.

[DG03] Donald A. Dawson and Andreas Greven. State dependent multitype spatial branching processes and their longtime behavior. Electron. J. Probab., 8(4):1-93, 2003.

[DGP11] Andrej Depperschmidt, Andreas Greven, and Peter Pfaffelhuber. Marked metric measure spaces. Electron. Commun. Probab., 16:174-188, 2011.

[DGP12] Andrej Depperschmidt, Andreas Greven, and Peter Pfaffelhuber. Tree-valued Fleming-Viot dynamics with mutation and selection. Ann. Appl. Probab., 22(6):2560-2615, 2012.

[DP91] Donald A. Dawson and Edwin A. Perkins. Historical processes. Mem. Amer. Math. Soc., 93(454):iv+179, 1991.

[EK86] Stewart N. Ethier and Thomas G. Kurtz. Markov Processes. Characterization and Convergence. John Wiley \& Sons, Inc., New York, 1986.

[EW06] Steven N. Evans and Anita Winter. Subtree prune and regraft: A reversible real tree-valued Markov process. Ann. Probab., 34(3):918-961, 2006.

[FM04] Nicolas Fournier and Sylvie Méléard. A microscopic probabilistic description of a locally regulated population and macroscopic approximations. Ann. Appl. Probab., 14(4):1880-1919, 2004.

[G112] Patric K. Glöde. Dynamics of Genealogical Trees for Autocatalytic Branching Processes. PhD thesis, FAU Erlangen, http://www.min.math.fau.de/fileadmin/min/users/gloede/Dissertation/PatricKarlGloedeDissertation.pdf, 2012.

[GLW05] Andreas Greven, Vlada Limic, and Anita Winter. Representation theorems for interacting Moran models, interacting Fisher-Wright diffusions and applications. Electron. J. Probab., 10(39):1286-1358, 2005.

$\left[\mathrm{GPG}^{+}\right.$04] Bryan T. Grenfell, Oliver G. Pybus, Julia R. Gog, James L. N. Wood, Janet M. Daly, Jenny A. Mumford, and Edward C. Holmes. Unifying the Epidemiological and Evolutionary Dynamics of Pathogens. Science, 303:327-332, 2004. (DOI: 10.1126/science.1090727).

[GPW09] Andreas Greven, Peter Pfaffelhuber, and Anita Winter. Convergence in distribution of random metric measure spaces ( $\Lambda$-coalescent measure trees). Probab. Theory Related Fields, 145:285-322, 2009.

[GPW13] Andreas Greven, Peter Pfaffelhuber, and Anita Winter. Tree-valued resampling dynamics: Martingale problems and applications. Probab. Theory Related Fields, 155:789-838, 2013.

[Gro99] Misha Gromov. Metric structures for Riemannian and non-Riemannian spaces, volume 152 of Progr. Math. Birkhäuser Boston Inc., Boston, MA, 1999. 
[GSW16] Andreas Greven, Rongfeng Sun, and Anita Winter. Continuum space limit of the genealogies of interacting Fleming-Viot processes on Z. Electron. J. Probab., 21(58):1-64, 2016.

[KL15] Sandra Kliem and Wolfgang Löhr. Existence of mark functions in marked metric measure spaces. Electron. J. Probab., 20(73):1-24, 2015.

[Kli14] Sandra Kliem. A compact containment result for nonlinear historical superprocess approximations for population models with trait-dependence. Electron. J. Probab., 19(97):1-13, 2014.

[LO07] Marc Lipsitch and Justin J. O'Hagan. Patterns of antigenic diversity and the mechanisms that maintain them. J. R. Soc. Interface, 4:787-802, 2007.

[Löh13] Wolfgang Löhr. Equivalence of Gromov-Prohorov- and Gromov's $\square_{\lambda}$-metric on the space of metric measure spaces. Electron. Commun. Probab., 18(17):1-10, 2013.

[LS09] Thomas M. Liggett and Rinaldo B. Schinazi. A stochastic model for phylogenetic trees. J. Appl. Probab., 46(2):601-607, 2009.

[MT12] Sylvie Méléard and Viet Chi Tran. Nonlinear historical superprocess approximations for population models with past dependence. Electron. J. Probab., 17(47):1-32, 2012.

[Pio10] Sven Piotrowiak. Dynamics of Genealogical Trees for Type- and State-dependent Resampling Models. PhD thesis, FAU Erlangen, https://opus4.kobv.de/opus4fau/files/1533/SvenPiotrowiakDissertation.pdf, 2010.

Sandra Kliem, Fakultät für Mathematik, Universität Duisburg-Essen, Thea-Leymann-Str. 9, 45127 Essen, Germany

E-mail address: sandra.kliem@uni-due.de

Anita Winter, Fakultät für Mathematik, Universität Duisburg-Essen, Thea-Leymann-Str. 9, 45127 Essen, Germany

E-mail address: anita.winter@uni-due.de 